\title{
Large time behavior of weakly coupled systems of first-order Hamilton-Jacobi equations
}

Fabio Camilli, Olivier Ley, Paola Loreti and Vinh Duc Nguyen

\begin{abstract}
We show a large time behavior result for class of weakly coupled systems of first-order Hamilton-Jacobi equations in the periodic setting. We use a PDE approach to extend the convergence result proved by Namah and Roquejoffre (Commun. Partial. Differ. Equ. 24(5-6):883-893, 1999) in the scalar case. Our proof is based on new comparison, existence and regularity results for systems. An interpretation of the solution of the system in terms of an optimal control problem with switching is given.
\end{abstract}

Mathematics Subject Classification (2000). Primary 49L25; Secondary 35F 30 and 35B25 and 58J37.

Keywords. Hamilton-Jacobi equations, Weakly coupled system, Large time behavior, Critical value.

\section{Introduction}

The aim of this paper is to study the large time behavior of the system of Hamilton-Jacobi equations

$$
\left\{\begin{array}{l}
\frac{\partial u_{i}}{\partial t}+H_{i}\left(x, D u_{i}\right)+\sum_{j=1}^{m} d_{i j}(x) u_{j}=0(x, t) \in \mathbb{T}^{N} \times(0,+\infty), \\
i=1, \ldots, m, \\
u_{i}(x, 0)=u_{0 i}(x) \quad x \in \mathbb{T}^{N},
\end{array}\right.
$$

where $\mathbb{T}^{N}$ is the $N$-dimensional torus. The Hamiltonians $H_{i}(x, p)$ are of eikonal type and the coupling is linear and monotone, i.e.,

$$
d_{i i}(x) \geq 0, \quad d_{i j}(x) \leq 0 \quad \text { for } \quad i \neq j \quad \text { and } \quad \sum_{j=1}^{m} d_{i j}(x) \geq 0, \quad \text { for all } x \in \mathbb{T}^{N} .
$$


The corresponding problem for the Hamilton-Jacobi equation

$$
\frac{\partial u}{\partial t}+H(x, D u)=0
$$

has been extensively investigated using both PDE methods, see Namah and Roquejoffre [24], Barles and Souganidis [4], and a dynamical approach: Fathi $[11,12]$, Roquejoffre [25], Davini and Siconolfi [9]. Some of these results have been also extended beyond the periodic setting: Barles and Roquejoffre [3], Ishii [18], Ichihara and Ishii [15] and for problems with periodic boundary conditions: see for instance Mitake [21-23]. We refer also the readers to Ishii $[17,19]$ for an overview.

In these works, one of the main result is that there exists a constant $c \in \mathbb{R}$, the so-called critical value or ergodic constant, and a solution $v$ of the stationary equation

$$
H(x, D u)=c
$$

such that

$$
u(x, t)+c t \rightarrow v(x) \quad \text { uniformly as } t \rightarrow+\infty .
$$

There are several equivalent characterizations of the critical value (see $[4,12]$ ), for example $c$ is the unique constant such that

$$
\frac{u(x, t)}{t} \rightarrow-c \quad \text { uniformly as } t \rightarrow+\infty,
$$

or

$$
c=\min \{a \in \mathbb{R}: H(x, D u)=a \text { has a subsolution }\} .
$$

While $c$ is uniquely determined, the main difficulty in proving a result like (1.5) is that (1.4) does not admit a unique solution (at least, the equation is invariant by addition of constants).

To our knowledge, there are not only no results of asymptotic type for the system (1.1), but also the study of corresponding ergodic problem

$$
H_{i}\left(x, D v_{i}(x)\right)+\sum_{j=1}^{m} d_{i j}(x) v_{j}(x)=c_{i} \quad x \in \mathbb{T}^{N},
$$

is not well understood (see [7] for some preliminary results).

With the aim of understanding if some convergence like (1.5) holds in the case of systems, we focus on the setting of Namah and Roquejoffre [24]. Let us start by recalling the main result of [24]. It takes place in the periodic setting and they assume that $H$ in (1.3) is continuous and of the type

$$
H(x, p)=F(x, p)-f(x) \quad x \in \mathbb{T}^{N}, p \in \mathbb{R}^{N},
$$

where $F$ is coercive and convex with respect to $p$. Besides, $F(x, p) \geq F(x, 0)=$ 0 . The function $f$ is continuous and satisfies

$$
f \geq 0 \quad \text { and } \quad \mathcal{F}_{\text {scalar }}=\left\{x \in \mathbb{T}^{N}: f(x)=0\right\} \neq \emptyset .
$$

It is simple to see by the characterization in (1.7) that $c=0$. Moreover by classical results in viscosity solution theory, $\mathcal{F}_{\text {scalar }}$ is a uniqueness set for (1.4), i.e. the solution of (1.4) is uniquely characterized by its value on this set. 
The coercitivity of the Hamiltonian provides the compactness of the functions $u(\cdot, t)$ for $t>0$. Then employing the semi-relaxed limits, one can pass to the limit and obtain the convergence result if one can prove the convergence of $u(\cdot, t)$ on the set $\mathcal{F}_{\text {scalar }}$. This latter result follows from the observation that, since $F(x, p) \geq 0$, a solution of (1.3) satisfies

$$
\frac{\partial u}{\partial t} \leq 0 \quad \text { on } \quad \mathcal{F}_{\text {scalar }} \times(0,+\infty) .
$$

Hence $u(\cdot, t)$ is nonincreasing and therefore converges uniformly on $\mathcal{F}_{\text {scalar }}$ and one concludes that it converges in all $\mathbb{T}^{N}$.

Our purpose is to reproduce the previous proof and therefore we assume that the Hamiltonians $H_{i}$ 's in (1.1) are as in [24] and the coupling matrix $D(x)=\left(d_{i j}\right)_{1 \leq i, j \leq m}$ satisfies (1.2). Under these assumptions, (1.1) has a unique viscosity solution in $\mathbb{T}^{N} \times[0,+\infty)$ for any continuous initial data $u_{0}: \mathbb{T}^{N} \rightarrow \mathbb{R}^{m}$.

For the simplicity of the exposition in this introduction, we assume moreover that

$$
\sum_{j=1}^{m} d_{i j}(x)=0, \quad i=1, \ldots, m, \quad \sum_{i=1}^{m} d_{i j}(x)=0, \quad j=1, \ldots, m,
$$

for $x \in \mathbb{T}^{N}$. Note that this assumption is not necessary. We can avoid it by using the results of Sect. 2 .

To continue, we have to understand what plays the role of $\mathcal{F}_{\text {scalar }}$ in (1.9) for systems. In the scalar case, there are important interpretations of the convergence (1.5) in terms of dynamical systems or optimal control theory [12]. Indeed, (1.5) means that the optimal trajectories of the related control problem are attracted by the set $\mathcal{F}_{\text {scalar }}$ where the running cost $f$ is 0 .

In the case of systems, the solutions $u_{i}$ 's of (1.1) are value functions of a piecewise deterministic optimal control problem with random switchings. The switchings are governed by a continuous in time Markov process with state space $\{1, \ldots, m\}$ and probability transitions from the mode $i$ to $j$ given by $\gamma_{i j}=-d_{i j}$ for $i \neq j$. See Sect. 6 for further details. A natural assumption to obtain the convergence of the value functions $u_{i}$ 's is to require that all the running costs $f_{i}$ 's vanish at least at some common point. It suggests that the optimal strategy consists in driving the trajectories to such a point where the running costs are 0 whatever the switchings will be (note that the coercivity of the Hamiltonian implies the controllability of the trajectories). So we introduce and assume that

$$
\mathcal{F}:=\bigcap_{i=1}^{m}\left\{x \in \mathbb{T}^{N}: f_{i}(x)=0\right\}=\left\{x \in \mathbb{T}^{N}: \sum_{i=1}^{m} f_{i}(x)=0\right\} \neq \emptyset
$$

(recall that the $f_{i} \geq 0$ ). We need an additional assumption on the coupling matrix, namely that $D(x)$ is irreducible, see Definition 2.1. Roughly speaking, it means that the coupling is not trivial and the system cannot be reduced to several subsystems of lower dimensions. 
The next step is to understand well the limit problem (1.8). Under the previous assumptions, $\mathcal{F}$ appears to be a uniqueness set for the stationary system (1.8) with $c_{i}=0$ (as in [24], we will prove that our assumptions imply that the critical value is $c=0)$. More precisely, on this set it is sufficient to control the value of the sum $v_{1}+\cdots+v_{m}$ of a solution to (1.8), see Theorem 3.3, a condition which seems to be new with respect to standard assumptions on weakly coupled systems $[10,20]$. Let us mention that, when (1.10) does not hold, we need to replace $\mathcal{F}$ with another set $\mathcal{A}$, see (3.6)-(3.7).

We then solve the so-called ergodic problem, which consists in finding a couple $(c, v) \in \mathbb{R}^{m} \times C\left(\mathbb{T}^{N} ; \mathbb{R}^{m}\right)$ of solutions to (1.8). The motivation comes from the formal expansion suggested by the convergence result of type (1.5) we are expecting for (1.1). Plugging $u_{\infty}(x)-c t \approx u(x, t)$ in (1.1), we obtain that $\left(c, u_{\infty}\right)$ should be a solution of

$$
H_{i}\left(x, D\left(u_{\infty}\right)_{i}\right)+\sum_{j=1}^{m} d_{i j}(x)\left(u_{\infty}\right)_{j}(x)-t \sum_{j=1}^{m} d_{i j}(x) c_{j}=c_{i},
$$

for all $i$, with $c \in \operatorname{ker}(D(x))$ to cancel the term in $t$. In Theorem 4.2, we prove the existence of a solution where $c \in \operatorname{ker}(D(x))$ and $v$ Lipschitz continuous on $\mathbb{T}^{N}$. The Lipschitz continuity of $v$ is an easy consequence of the coercivity of the $F_{i}$ 's. Under the assumptions (1.10), it is easy to see that $\operatorname{ker}(D(x))$ reduces to the line spanned by $(1, \ldots, 1)$ so $c=\left(c_{1}, \ldots, c_{1}\right)$. Moreover, due to (1.11), we obtain that $c=(0, \ldots, 0)$ is uniquely determined.

At this step, it is worth noticing that we can solve the ergodic problem in a more general setting (see Theorem 4.3), in particular without assuming (1.11). We obtain the following condition on $c$,

$$
c=\left(c_{1}, \ldots, c_{1}\right) \quad \text { and } \quad \sum_{i=1}^{m} \min _{\mathbb{T}^{N}} f_{i}(x) \leq-c_{1} \leq \min _{\mathbb{T}^{N}} \sum_{i=1}^{m} f_{i}(x) .
$$

This gives again an indication that assuming $\mathcal{F} \neq \emptyset$ is a first natural case to consider, since, in this case, inequalities are replaced with equalities in the above formula and the ergodic constant is univocally defined.

We are now in force to consider the large time result (Theorem 5.4). The coercivity of the Hamiltonians and the existence of a solution to the ergodic problem give the compactness of the sequences $u_{i}$ 's in $W^{1, \infty}\left(\mathbb{T}^{N} \times[0,+\infty)\right)$. An easy consequence is the convergence (1.6) for all $i$ to 0 (since $c=0$ in our case). To mimic the proof of [24], we need to prove the convergence of the $u_{i}$ 's on $\mathcal{F}$. This is the most difficult part of the work.

Indeed, by summing the equations (1.1) for $i=1, \ldots, m$, we obtain

$$
\sum_{i=1}^{m} \frac{\partial u_{i}}{\partial t}+\sum_{i=1}^{m} H_{i}\left(x, D u_{i}\right)+\sum_{i, j=1}^{m} d_{i j} u_{j}=0
$$

Using that $H_{i}\left(x, D u_{i}\right) \geq 0$ on $\mathcal{F}$ and (1.10), we obtain easily that

$$
\frac{\partial}{\partial t} \sum_{i=1}^{m} u_{i}(x, t) \leq 0 \quad \text { on } \mathcal{F}
$$


and therefore $t \mapsto\left(u_{1}+\cdots+u_{m}\right)(\cdot, t)$ is nonincreasing and converges uniformly as $t \rightarrow+\infty$ on $\mathcal{F}$. But this is not enough to prove the convergence of each $u_{i}$ on $\mathcal{F}$.

To overcome this difficulty, we use some ideas of [4]. We choose a subsequence $t_{n} \rightarrow+\infty$ such that $u\left(\cdot, t_{n}+\cdot\right)$ converges uniformly to some $w(\cdot, \cdot)$ in $W^{1, \infty}\left(\mathbb{T}^{N} \times[0,+\infty)\right)$. By stability of the viscosity solutions, $w$ is still solution of (1.1) and we earn something: now, $t \mapsto\left(w_{1}+\cdots+w_{m}\right)(\cdot, t)$ is constant on $\mathcal{F}$. Therefore (1.13) holds for the $w_{i}$ 's with an equality. It follows from (1.12) that

$$
\sum_{i=1}^{m} H_{i}\left(x, D w_{i}\right)=0 \quad \text { on } \mathcal{F} \times(0,+\infty)
$$

Since $H_{i}=F_{i} \geq 0$ on $\mathcal{F}$, we infer that $H_{i}\left(x, D w_{i}\right)=0$ for all $1 \leq i \leq m$. Therefore, the system (1.1) reduces to a linear differential system

$$
\frac{\partial w}{\partial t}(x, t)+D(x) w(x, t)=0 \quad t \geq 0,
$$

for every $x \in \mathcal{F}$. Using that $D(x)$ satisfies (1.2), (1.10) and is irreducible, we can prove the convergence of each $w_{i}(\cdot, t)$ on $\mathcal{F}$ and then on $\mathbb{T}^{N}$ by applying the comparison Theorem 3.3. The conclusion follows by proving that $u(\cdot, t)$ converges to the same limit as $w(\cdot, t)$.

The paper is organized as follows. In Sect. 2, we study some properties of the coupling matrix $D$ without assumption (1.10). Section 3 is devoted to study existence and uniqueness of the stationary problem. In Sect. 4 we solved the ergodic problem. The convergence result is proved in Sect. 5. Finally, in Sect. 6, we give a control theoretic interpretation of the problem.

We learnt recently that H. Mitake and H.V. Tran (In preparation) studied systems of two equations $(m=2)$ both in our setting (see Remark $5.7(3)$ ) and also in some particular cases related to [4].

Notation. If $p=\left(p_{1}, \ldots, p_{m}\right)$ is a vector in $\mathbb{R}^{m}$, then $p \geq 0$ (respectively $p>0$ ) means that $p_{i} \geq 0$ (respectively $p_{i}>0$ ) for $1 \leq i \leq m$.

\section{Preliminaries on coupling matrices}

We consider the matrix $D(x)=\left(d_{i j}(x)\right)_{1 \leq i, j \leq m}$ and call it a coupling matrix for the systems (1.1) and (1.8). We assume that the coupling matrices satisfy the following standard assumptions (see [16,20])

$$
\begin{aligned}
& d_{i j}: \mathbb{T}^{N} \rightarrow \mathbb{R} \text { are continuous and, for all } x \in \mathbb{T}^{N}, \\
& d_{i i}(x) \geq 0, \quad d_{i j}(x) \leq 0 \text { for } i \neq j \text { and } \sum_{j=1}^{m} d_{i j}(x) \geq 0 .
\end{aligned}
$$


We introduce some conditions on the matrix $D$ we will be interested in:

Definition 2.1. We say that $D$ is

(i) a $M$-matrix if

$D=s I-B, \quad$ for some $s>0, \quad B=\left(b_{i j}\right)_{1 \leq i, j \leq m}, \quad b_{i j} \geq 0$,

with $s \geq \rho(B)$ and $\rho(B)$ the spectral radius of $B$,

( $I$ is the identity matrix).

(ii) irreducible if, for all subset $\mathcal{I} \varsubsetneqq\{1, \ldots, m\}$ then there exists $i \in \mathcal{I}$ and $j \notin \mathcal{I}$ such that $d_{i j} \neq 0$.

Lemma 2.2. If $D$ satisfies (2.1), then it is a $M$-matrix for any $x$.

Proof. If $D=0$, there is nothing to prove. Otherwise, $s:=\max _{1 \leq k \leq m} d_{k k}>0$. Then, we can write $D=s I-B$ with $B=\left(b_{i j}\right)$ with $b_{i i}=s-d_{i i}$ and $b_{i j}=-d_{i j}$ for $i \neq j$. Since $B \geq 0$, by Perron-Froebenius theorem, the spectral radius $\rho(B)$ of $B$ is an eigenvalue and there exists a nonnegative eigenvector $p$ such that $B p=\rho(B) p$. Therefore $D p=(s-\rho(B)) p$. Let $p_{k}=\max _{i} p_{i}$. Since $B \neq 0$, we have $p_{k}>0$ and, using that $d_{i j} \leq 0$ for $i \neq j$,

$$
0 \leq\left(\sum_{j=1}^{m} d_{k j}\right) p_{k} \leq \sum_{j=1}^{m} d_{k j} p_{j}=(s-\rho(B)) p_{k}
$$

and we conclude that $s \geq \rho(B)$.

Let us give a characterization of an irreducible matrix.

Lemma 2.3. $D$ is irreducible if and only if: for all $i, j \in\{1, \ldots, m\}$, there exists $n \in \mathbb{N}$ and a sequence $i_{0}=i, i_{1}, i_{2}, \ldots, i_{n}=j$ such that $d_{i_{l-1} i_{l}} \neq 0$ for all $1 \leq l \leq n$ (in this case we say that there exists a chain between $i$ and $j$ ).

Proof. Let $i \in\{1, \ldots, m\}$ and $\mathcal{I}_{i}$ be the subset of $\{1, \ldots, m\}$ containing all the chains starting from $i$. It is obvious that, if $D$ is irreducible, then $\mathcal{I}_{i}=$ $\{1, \ldots, m\}$. Conversely, let $\mathcal{I} \varsubsetneqq\{1, \ldots, m\}, i \in \mathcal{I}$ and $j \notin \mathcal{I}$. By assumption, there exists a chain $i_{0}=i, i_{1}, \ldots, i_{n}=j$ between $i$ and $j$. Let $1 \leq \bar{l} \leq n$ be the smallest $l$ such that $l \notin \mathcal{I}$. Then $d_{i_{\bar{l}-1} i_{\bar{l}}} \neq 0$.

Example 2.4. If $d_{i j} \neq 0$ for all $i, j$ then it is obvious that $D$ is irreducible. In particular, when $D$ satisfies (2.1) and, in addition, $d_{i j}<0$ for $i \neq j$, then it is irreducible. The matrices

$$
\left[\begin{array}{ccc}
1 & -1 & 0 \\
0 & 1 & -1 \\
-1 & 0 & 1
\end{array}\right] \text { and }\left[\begin{array}{cc}
\alpha & -\alpha \\
-\beta & \beta
\end{array}\right] \text { with } \alpha, \beta>0
$$

are irreducible and satisfy (2.1). On the contrary,

$$
\left[\begin{array}{cccc}
1 & -1 & 0 & 0 \\
-1 & 1 & 0 & 0 \\
0 & 0 & 1 & -1 \\
0 & 0 & -1 & 1
\end{array}\right] \text { and }\left[\begin{array}{cc}
0 & 0 \\
-1 & 1
\end{array}\right]
$$

satisfy (2.1) but are not irreducible. 
Remark 2.5. For $M$-matrices and irreducible $M$-matrices, see [5, Chapter 6] and [26]. These assumptions are natural when studying coupled systems of partial differential equations like (3.1) and (5.1). To expect some maximum principles, one usually needs $M$-matrices and, roughly speaking, when the coupling matrix is irreducible, it means that the equations are coupled in a non trivial way. For instance, in the cases (2.2), the system of equations is decoupled (into two subsets of equations) and is triangular, respectively. We refer to the work of Busca and Sirakov [6] and the references therein for details.

Lemma 2.6. Let $E_{1}, E_{2} \subset \mathbb{T}^{N}$ be closed subsets, let $E=E_{1} \cap E_{2}$, suppose that $D(x)=\left(d_{i j}(x)\right)_{1 \leq i, j \leq m}$ is such that (2.1) holds and

$$
\begin{aligned}
& \sum_{j=1}^{m} d_{i j}(x)=0 \text { for } x \in E_{1}, \quad i=1, \ldots, m . \\
& D(x) \text { is irreducible for } x \in E_{2},
\end{aligned}
$$

(i) For all $x \in E, D(x)$ is degenerate of rank $m-1, \operatorname{ker}(D(x))=$ $\operatorname{span}\{(1, \ldots, 1)\}$ and the real part of each nonzero complex eigenvalue of $D(x)$ is positive. Moreover there exists a positive continuous function $\Lambda=\left(\Lambda_{1}, \ldots, \Lambda_{m}\right): E \rightarrow \mathbb{R}^{m}$ such that $\Lambda(x)>0$ and $D(x)^{T} \Lambda(x)=0$ for all $x \in E$.

(ii) In the set $E_{2} \backslash E_{1}$, where one only has $\sum_{j=1}^{m} d_{i j}(x) \geq 0$, then there exists a positive continuous function $\Lambda=\left(\Lambda_{1}, \ldots, \Lambda_{m}\right): E_{2} \backslash E_{1} \rightarrow \mathbb{R}^{m}$ such that $\Lambda(x)>0$ and $D(x)^{T} \Lambda(x) \geq 0$ for all $x \in E_{2} \backslash E_{1}$.

Proof. (i) At first, we fix any $x \in E$ (this part of the proof comes from [5, p. 156]). From $(2.3),(1, \ldots, 1) \in \operatorname{ker}(D(x))$. From Lemma 2.2 and $(2.4), D(x)$ is a nonzero $M$-matrix so we can write $D(x)=s(x) I-B(x), s(x)>0$, $s(x) \geq \rho(B(x))$ and $B(x) \geq 0$. It is obvious that, if $D(x)$ is irreducible, then so is $B(x)$. By Perron-Froebenius theorem, it follows that $\rho(B(x))$ is a simple eigenvalue of $B(x)$. Since, $D(x)(1, \ldots, 1)=0=s(x)(1, \ldots, 1)-B(x)(1, \ldots, 1)$, we have $s(x)=\rho(B(x))$ and 0 is a simple eigenvalue of $D(x)$. Thus $D(x)$ has rank $m-1$. Besides, if $\lambda \in \mathbb{C} \backslash\{0\}$ is another eigenvalue of $D(x)$, then $s(x)-\lambda$ is an eigenvalue of $B(x)$. It follows that $|s(x)-\lambda| \leq \rho(B(x))=s(x)$ and, since $\lambda \neq 0$, the real part of $\lambda$ must be positive.

Since $B^{T}(x)$ is also an irreducible nonnegative matrix with $\rho(B(x))=$ $\rho\left(B^{T}(x)\right)$, using again Perron-Froebenius theorem, we obtain the existence of an eigenvector $\Lambda(x)>0$ such that $B^{T}(x) \Lambda(x)=\rho(B(x)) \Lambda(x)$. Therefore $D^{T}(x) \Lambda(x)=0$.

Then, we shall prove that it is possible to choose $\Lambda(x)$ continuously. Let $\operatorname{com}(D(x))$ be the cofactor matrix of $D(x)$. Since $D(x)$ is non invertible, we have $D(x)^{T} \operatorname{com}(D(x))=0$. Therefore the columns $C_{j}(x), j=1, \ldots, m$, of $\operatorname{com}(D(x))$ are in the kernel of $D(x)^{T}$. From the first part of the proof, we obtain that there exist functions $\lambda_{i}: E \rightarrow \mathbb{R}$ such that $C_{j}(x)=\lambda_{j}(x) \Lambda(x)$. Define $\left|C_{j}\right|(x)$ as the absolute value of the coefficients of $C_{j}(x)$ and 


$$
\tilde{\Lambda}(x)=\sum_{j=1}^{m}\left|C_{j}\right|(x)=\left(\sum_{j=1}^{m}\left|\lambda_{j}(x)\right|\right) \Lambda(x) .
$$

On one hand, $\tilde{\Lambda}(x)>0$ since $\operatorname{com}(D(x))$ is not 0 . On the other hand, the continuity of the coefficients of $D(x)$ implies the continuity of the coefficients of $\operatorname{com}(D(x))$ and therefore the maps $x \mapsto\left|C_{j}\right|(x)$ are continuous on $E$. We conclude that $\tilde{\Lambda}$ is continuous.

(ii) The proof is an easy consequence of (i). Define $\alpha_{i}(x):=\sum_{j=1}^{m} d_{i j}(x) \geq 0$, the diagonal matrix $\Delta(x):=\operatorname{diag}\left(\alpha_{1}(x), \ldots, \alpha_{m}(x)\right)$ and $\tilde{D}:=D-\Delta$. It is straightforward that $\tilde{D}$ is still an irreducible $M$-matrix such that (2.3) holds on $E_{2} \backslash E_{1}$. By (i), there exists a continuous $\Lambda: E_{2} \backslash E_{1} \rightarrow \mathbb{R}^{m}$ such that $\Lambda(x)>0$ and $\tilde{D}(x)^{T} \Lambda(x)=0$. It follows $D(x)^{T} \Lambda(x)=\Delta(x)^{T} \Lambda(x) \geq 0$ by assumption. It completes the proof.

\section{Comparison, existence and regularity for the stationary system}

In this section we study existence and uniqueness of the solution to the stationary system

$$
H_{i}\left(x, D u_{i}\right)+\sum_{j=1}^{m} d_{i j}(x) u_{i}=0 \quad \text { in } \mathbb{T}^{N}, \quad 1 \leq i \leq m,
$$

where $H_{i}: \mathbb{T}^{N} \times \mathbb{R}^{N} \rightarrow \mathbb{R}, i=1, \ldots, m$, is a continuous function which takes the form

$$
H_{i}(x, p)=F_{i}(x, p)-f_{i}(x) .
$$

We assume that, for all $i=1, \ldots, m$,

$f_{i}, F_{i}(\cdot, p)$ are continuous 1 -periodic for any $p \in \mathbb{R}^{N}$;

$F_{i}(x, \cdot)$ is convex, coercive and, for any $x \in \mathbb{T}^{N}, p \in \mathbb{R}^{N}, F_{i}(x, p) \geq F_{i}(x, 0)=0$;

$$
f_{i}(x) \geq 0 .
$$

We set for $i=1, \ldots, m$,

$$
\begin{gathered}
\mathcal{F}=\left\{x \in \mathbb{T}^{N}: \sum_{i=1}^{m} f_{i}(x)=0\right\}, \quad \mathcal{D}_{i}=\left\{x \in \mathbb{T}^{N}: \sum_{j=1}^{m} d_{i j}(x)=0\right\} \\
\mathcal{A}=\mathcal{F} \cap\left(\bigcap_{i=1}^{m} \mathcal{D}_{i}\right)
\end{gathered}
$$

Remark 3.1. Note that, under (3.5), if $\mathcal{F}$ is not empty, it means that all the $f_{i}$ 's achieve a common minimum 0 at some common point.

We recall the definition of viscosity solutions for the system (3.1) (see $[16,20]$ for more details about systems of Hamilton-Jacobi equations). Let $U S C$ (respectively $L S C$ ) denotes the upper-semicontinuous (respectively lower-semicontinuous) functions. 
Definition 3.2. (i) An $U S C$ function $u: \mathbb{R}^{N} \rightarrow \mathbb{R}^{m}$ is said a viscosity subsolution of (3.1) if whenever $\phi \in C^{1}, i \in\{1, \ldots, m\}$ and $u_{i}-\phi$ attains a local maximum at $x$, then

$$
H_{i}(x, D \phi(x))+\sum_{j=1}^{m} d_{i j}(x) u_{j}(x) \leq 0 .
$$

(ii) A $L S C u: \mathbb{R}^{N} \rightarrow \mathbb{R}^{m}$ is said a viscosity supersolution of (3.1) if whenever $\phi \in C^{1}, i \in\{1, \ldots, m\}$ and $u_{i}-\phi$ attains a local minimum at $x$, then

$$
H_{i}(x, D \phi(x))+\sum_{j=1}^{m} d_{i j}(x) u_{j}(x) \geq 0 .
$$

(iii) A continuous function $u$ is said a viscosity solution of (3.1) if it is both a viscosity sub- and supersolution of (3.1).

We first prove a comparison theorem for (3.1) giving a boundary condition on the set (3.7), which turns out to be a uniqueness set for the system.

Theorem 3.3. Assume (2.1) and (3.3)-(3.5). Let $u \in U S C\left(\mathbb{T}^{N}\right)$ and $v \in$ $L S C\left(\mathbb{T}^{N}\right)$ be respectively a bounded subsolution and a bounded supersolution of (3.1) and suppose that one of the following set of assumptions holds:

(i) Classical case:

$$
\sum_{j=1}^{m} d_{i j}(x)>0 \quad \text { in } \mathbb{T}^{N} \text { for all } 1 \leq i \leq m .
$$

(ii) Degenerate case: Assume (2.4) holds with $E_{2}=\mathbb{T}^{N}$ and there exists

$$
\begin{aligned}
& \Lambda: \mathcal{A} \rightarrow \mathbb{R}^{m}, \Lambda \geq 0, \sum_{i=1}^{m} \Lambda_{i}>0, \text { such that } \\
& \sum_{i=1}^{m} \Lambda_{i}(x) u_{i}(x) \leq \sum_{i=1}^{m} \Lambda_{i}(x) v_{i}(x), x \in \mathcal{A} .
\end{aligned}
$$

Then

$$
u \leq v \quad \text { in } \mathbb{T}^{N}
$$

Proof. The proof of the classical case can be deduced from the lines of the degenerate case, so we skip it and turn to the degenerate case. See some comments at the end of Case 2 below.

Let $0<\mu<1$, and consider

$$
\sup _{\mathbb{T}^{N}} \sup _{1 \leq k \leq m}\left\{\mu u_{k}-v_{k}\right\}=: M_{\mu}
$$

We assume that $M_{\mu}>0$ (otherwise, there is nothing to prove). By compactness, the above maximum is achieved for some $k_{0}$ at some $x_{0} \in \mathbb{T}^{N}$. We set

$$
\mathcal{I}=\left\{k \in\{1, \ldots, m\}:\left(\mu u_{k}-v_{k}\right)\left(x_{0}\right)=M_{\mu}\right\}
$$

We distinguish 3 cases. 
Case 1. $\mathcal{I}=\{1, \ldots, m\}$ and $x_{0} \in \mathcal{A}$. For all $k$, we get

$$
\begin{aligned}
\sum_{k=1}^{m} \Lambda_{k}\left(x_{0}\right) M_{\mu} & =\sum_{k=1}^{m} \Lambda_{k}\left(x_{0}\right)\left(\mu u_{k}-v_{k}\right)\left(x_{0}\right) \leq(\mu-1) \sum_{k=1}^{m} \Lambda_{k}\left(x_{0}\right) v_{k}\left(x_{0}\right) \\
& \leq(1-\mu) \sum_{k=1}^{m} \Lambda_{k}\left(x_{0}\right)|v|_{\infty}
\end{aligned}
$$

and therefore $M_{\mu} \leq(1-\mu)|v|_{\infty}$.

Case 2. $\mathcal{I}=\{1, \ldots, m\}$ but $x_{0} \notin \mathcal{A}$. One can find $i \in\{1, \ldots, m\}$ such that

$$
\text { either } \quad f_{i}\left(x_{0}\right)>0 \text { or } \quad \sum_{j=1}^{m} d_{i j}\left(x_{0}\right)>0 \text {. }
$$

Consider

$$
\sup _{\mathbb{T}^{N} \times \mathbb{T}^{N}}\left\{\mu u_{i}(x)-v_{i}(y)-\frac{|x-y|^{2}}{2 \epsilon^{2}}-\left|x-x_{0}\right|^{2}\right\} .
$$

The latter maximum is greater than $M_{\mu}$ and is achieved at some $(\bar{x}, \bar{y})$ which satisfy the following classical properties:

$$
\bar{x}, \bar{y} \rightarrow x_{0} \quad \text { and } \quad \frac{|\bar{x}-\bar{y}|^{2}}{2 \epsilon^{2}},\left|\bar{x}-x_{0}\right|^{2} \rightarrow 0 \quad \text { as } \epsilon \rightarrow 0 .
$$

We set $p_{\epsilon}=\frac{\bar{x}-\bar{y}}{\epsilon^{2}}$.

Writing that $u_{i}$ is a viscosity subsolution of (3.1), we have

$$
\mu F_{i}\left(\bar{x}, \frac{p_{\epsilon}+2\left(\bar{x}-x_{0}\right)}{\mu}\right)+\sum_{j=1}^{m} d_{i j}(\bar{x}) \mu u_{j}(\bar{x}) \leq \mu f_{i}(\bar{x})
$$

and writing that $v_{i}$ is a supersolution of (3.1), we get

$$
F_{i}\left(\bar{y}, p_{\epsilon}\right)+\sum_{j=1}^{m} d_{i j}(\bar{y}) v_{j}(\bar{y}) \geq f_{i}(\bar{y}) .
$$

From the coercivity of $F_{i}$ and the boundedness of $f_{i}$ and the $d_{i j}$ 's on $\mathbb{T}^{N}$, (3.14) implies that

$$
\left|p_{\epsilon}\right| \leq C=C\left(F_{i}, D, f_{i}\right) .
$$

We subtract the two inequalities. At first

$$
\begin{aligned}
\mu F_{i}\left(\bar{x}, \frac{p_{\epsilon}+2\left(\bar{x}-x_{0}\right)}{\mu}\right)-F_{i}\left(\bar{y}, p_{\epsilon}\right)= & \mu F_{i}\left(\bar{x}, \frac{p_{\epsilon}+2\left(\bar{x}-x_{0}\right)}{\mu}\right)-F_{i}\left(\bar{x}, p_{\epsilon}\right) \\
& +F_{i}\left(\bar{x}, p_{\epsilon}\right)-F_{i}\left(\bar{y}, p_{\epsilon}\right) .
\end{aligned}
$$

By convexity of $F_{i}$, we have

$$
\begin{aligned}
& \mu F_{i}\left(\bar{x}, \frac{p_{\epsilon}+2\left(\bar{x}-x_{0}\right)}{\mu}\right)-F_{i}\left(\bar{x}, p_{\epsilon}\right) \\
& \geq-(1-\mu) F_{i}\left(\bar{x},-\frac{2\left(\bar{x}-x_{0}\right)}{1-\mu}\right) \underset{\epsilon \rightarrow 0}{\longrightarrow}-(1-\mu) F_{i}\left(x_{0}, 0\right)=0
\end{aligned}
$$


by (3.4). On the other hand, using (3.13) and the uniform continuity of $F_{i}$ on the compact subset $\mathbb{T}^{N} \times \bar{B}(0, C)$, where $C$ is given by $(3.15)$, we have

$$
\left|F_{i}\left(\bar{x}, p_{\epsilon}\right)-F_{i}\left(\bar{y}, p_{\epsilon}\right)\right| \leq o_{\epsilon}(1)
$$

where $o_{\epsilon}(1) \rightarrow 0$ as $\epsilon \rightarrow 0$. Moreover

$$
\begin{aligned}
\sum_{j=1}^{m} d_{i j}(\bar{x}) \mu u_{j}(\bar{x})-d_{i j}(\bar{y}) v_{j}(\bar{y})= & \sum_{j=1}^{m} d_{i j}(\bar{x})\left(\mu u_{j}(\bar{x})-v_{j}(\bar{y})\right) \\
& +\sum_{j=1}^{m}\left(d_{i j}(\bar{x})-d_{i j}(\bar{y})\right) v_{j}(\bar{y})
\end{aligned}
$$

and

$$
\sum_{j=1}^{m}\left(d_{i j}(\bar{x})-d_{i j}(\bar{y})\right) v_{j}(\bar{y}) \underset{\epsilon \rightarrow 0}{\longrightarrow} 0
$$

since $v_{i}$ are bounded and $d_{i j}$ is continuous. Finally, we obtain

$$
\sum_{j=1}^{m} d_{i j}(\bar{x})\left(\mu u_{j}(\bar{x})-v_{j}(\bar{y})\right) \leq(\mu-1) f_{i}(\bar{x})+o_{\epsilon}(1) .
$$

Since $\mu u_{j}-v_{j}$ is $U S C$, for all $j$,

$$
\limsup _{\epsilon \rightarrow 0}\left(\mu u_{j}(\bar{x})-v_{j}(\bar{y})\right) \leq \mu u_{j}\left(x_{0}\right)-v_{j}\left(x_{0}\right) \leq M_{\mu} .
$$

Recalling that $d_{i j} \leq 0$ for $j \neq i$, it follows

$$
d_{i j}(\bar{x})\left(\mu u_{j}(\bar{x})-v_{j}(\bar{y})\right) \geq d_{i j}(\bar{x}) M_{\mu}+o_{\epsilon}(1) \quad \text { for } j \neq i .
$$

Moreover, since $i \in \mathcal{I}$, we have $\mu u_{i}(\bar{x})-v_{i}(\bar{y}) \geq M_{\mu}$ and

$$
d_{i i}(\bar{x})\left(\mu u_{i}(\bar{x})-v_{i}(\bar{y})\right) \geq d_{i i}(\bar{x}) M_{\mu} .
$$

From (3.16), we get

$$
\left(\sum_{j=1}^{m} d_{i j}(\bar{x})\right) M_{\mu} \leq(\mu-1) f_{i}(\bar{x})+o_{\epsilon}(1)
$$

which leads to a contradiction from $\epsilon$ small enough since $M_{\mu}>0$ and, by (3.12), either $\sum_{j=1}^{m} d_{i j}\left(x_{0}\right)>0$ or $f_{i}\left(x_{0}\right)>0$.

The proof of the theorem in the classical case reduces to Case 2. Indeed, in the classical case, $\mathcal{F}=\emptyset$ and, regardless $\mathcal{I}=\{1, \ldots m\}$ or not, we can always choose $i \in \mathcal{I}$ in order that (3.12) holds. Notice that we do not need (3.5). It suffices to send $\epsilon \rightarrow 0$ and $\mu \rightarrow 1$ in (3.19). 
Case 3. $\mathcal{I} \neq\{1, \ldots m\}$. Using that $D\left(x_{0}\right)$ is irreducible, there exist $i \in \mathcal{I}$ and $k \notin \mathcal{I}$ such that $d_{i k}\left(x_{0}\right)<0$. We argue as in Case 2 to obtain (3.16). Inequalities (3.17) and (3.18) hold true in this case too. But we need a more precise estimate for the index $k$. Since $k \notin \mathcal{I}$,

$$
\limsup _{\epsilon \rightarrow 0}\left(\mu u_{k}(\bar{x})-v_{k}(\bar{y})\right) \leq \mu u_{k}\left(x_{0}\right)-v_{k}\left(x_{0}\right) \leq M_{\mu}-\eta \quad \text { for some } \eta>0 .
$$

From (3.16), (3.17), (3.18) and (3.20), we obtain

$$
d_{i i}(\bar{x}) M_{\mu}+\sum_{j \neq i, k} d_{i j}(\bar{x}) M_{\mu}+d_{i k}(\bar{x})\left(M_{\mu}-\eta\right) \leq(\mu-1) f_{i}(\bar{x})+o_{\epsilon}(1) .
$$

It follows

$$
-d_{i k}\left(x_{0}\right) \eta \leq\left(\sum_{j=1}^{m} d_{i j}(\bar{x})\right) M_{\mu}-d_{i k}\left(x_{0}\right) \eta \leq(\mu-1) f_{i}(\bar{x})+o_{\epsilon}(1) \leq o_{\epsilon}(1)
$$

which leads to a contradiction for small $\epsilon$ since $d_{i k}\left(x_{0}\right)<0$.

End of the proof. The only possible case is $M_{\mu} \leq(1-\mu)|v|_{\infty}$ which implies that $M_{1} \leq 0$. The proof is complete.

Remark 3.4. The classical case (3.8) corresponds to the scalar case $\lambda u+$ $H(x, D u)=0$ with $\lambda>0$ and, in this case, we always have existence and uniqueness of the solution. Theorem 3.3 (ii) says that $\mathcal{A}$ is an uniqueness set for (3.1). We recall that for the single equation $F(x, D u)=f(x)$, where $F, f$ satisfy (3.3)-(3.5), the uniqueness set is $\left\{x \in \mathbb{T}^{N}: f(x)=0\right\}$ (see Fathi [12]). Notice that $\mathcal{A}$ maybe empty so we have automatically comparison (but the existence of solutions may fail). When $\mathcal{A}$ is not empty, it is enough to assume $u_{i} \leq v_{i}$ for one $i$ (this is a consequence of the irreducibility of the coupling matrix).

Before giving an existence result for (3.1), we prove Lipschitz regularity of subsolutions.

Lemma 3.5. Assume that (2.1) and (3.3)-(3.5) hold. Let $u \in U S C\left(\mathbb{T}^{N}\right)$ be a bounded viscosity subsolution to (3.1). Then $u$ is Lipschitz continuous in $\mathbb{T}^{N}$ with a constant $L=L\left(H_{1}, \ldots, H_{m}, D,|u|_{\infty}\right)$. If $(2.3)-(2.4)$ hold with $E_{1}=$ $E_{2}=\mathbb{T}^{N}$, then $L$ is independent of $|u|_{\infty}$.

Proof. From the coercitivity of the Hamiltonians $F_{i}$ and [2, Lemma 2.5 p. 33], it is sufficient to prove that $u_{i}$ is a viscosity subsolution of $F_{i}\left(x, D u_{i}\right) \leq C$ in $\mathbb{T}^{N}$. We have

$$
F_{i}\left(x, D u_{i}\right) \leq \sum_{j=1}^{m}\left|d_{i j}(x)\right|\left|u_{j}(x)\right|+f_{i}(x),
$$

which gives the result with a Lipschitz constant depending on $H_{1}, \ldots, H_{m}$, $|D|_{\infty},|u|_{\infty}$

Now, if (2.3)-(2.4) hold in $\mathbb{T}^{N}$, then, from Lemma 2.6(i), there exists a continuous function $\Lambda: \mathbb{T}^{N} \rightarrow \mathbb{R}^{m}, \Lambda>0$, such that $D(x)^{T} \Lambda(x)=0$ 
for all $x \in \mathbb{T}^{N}$. By multiplying Equations (4.2) by $\Lambda_{i}(x)$ and summing for $i=1, \ldots, m$, we obtain

$$
\sum_{i=1}^{m} \Lambda_{i}(x) F_{i}\left(x, D u_{i}\right)+\sum_{i, j=1}^{m} \Lambda_{i}(x) d_{i j}(x) u_{j}=\sum_{i=1}^{m} \Lambda_{i}(x) f_{i}(x) .
$$

We have

$$
\sum_{i, j=1}^{m} \Lambda_{i}(x) d_{i j}(x) u_{j}=\sum_{j=1}^{m}\left(\sum_{i=1}^{m} \Lambda_{i}(x) d_{i j}(x)\right) u_{j}=0
$$

since $D(x)^{T} \Lambda(x)=0$. It follows from (3.21) that

$$
\sum_{i=1}^{m} \Lambda_{i}(x) F_{i}\left(x, D u_{i}\right) \leq \sum_{i=1}^{m}\left|\Lambda_{i} f_{i}\right|_{\infty},
$$

By the compactness of $\mathbb{T}^{N}$ and the continuity of $\Lambda$, there exists $\eta=\eta(D)>0$ such that $\Lambda_{i} \geq \eta$ on $\mathbb{T}^{N}$ for all $i$. It completes the proof.

To state an existence result with prescribed values on $\mathcal{A}$, we need to introduce some definitions of Fathi and Siconolfi [13]. Define, for every $x \in \mathbb{T}^{N}$, $p \in \mathbb{R}^{N}$,

$$
F(x, p)=\max _{1 \leq i \leq m} F_{i}(x, p) \text { and } \quad f(x)=\min _{1 \leq i \leq m} f_{i}(x)
$$

and set

$S(y, x)=\max \left\{u(x): u\right.$ subsolution of $F(x, D u) \leq f(x)$ on $\mathbb{T}^{N}$ with $\left.u(y)=0\right\}$.

Proposition 3.6. Assume (2.1), (2.4) with $E_{2}=\mathbb{T}^{N},(3.3)-(3.5)$.

(i) (Classical case) If (3.8) holds, then there exists a unique continuous viscosity solution of (3.1).

(ii) (Degenerate case) Suppose that (2.3) holds with $E_{1}=\mathbb{T}^{N}$ and $\mathcal{F} \neq \emptyset$. For any continuous function $g: \mathcal{F} \rightarrow \mathbb{R}$ satisfying

$$
g(x)\left(\sum_{i=1}^{m} \Lambda_{i}(x)\right)^{-1}-g(y)\left(\sum_{i=1}^{m} \Lambda_{i}(y)\right)^{-1} \leq S(y, x),
$$

there exists a unique continuous viscosity solution $u$ of (3.1) such that

$$
\sum_{i=1}^{m} \Lambda_{i}(x) u_{i}(x)=g(x) \quad x \in \mathcal{F}
$$

where the continuous function $\Lambda: \mathbb{T}^{N} \rightarrow \mathbb{R}^{m}, \Lambda>0$, is given by Lemma 2.6.

Remark 3.7. The assumption (3.23) can be seen as a compatibility condition. We cannot prescribe any function $g$ on $\mathcal{A}$. Indeed, for instance, if $\Lambda_{i}=1$ for all $i$ and $g$ has a large Lipschitz constant compared to the one given by Lemma 3.5, then it is straightforward to see that it is not possible to build a solution $u$ satisfying (3.24). 
Proof. The proof of the existence of a solution is based on Perron's method. We start by building subsolutions and supersolutions.

From $(3.4),(3.5)$ and $(2.1)$, we obtain that $\psi_{C}=(C, \ldots, C)$ is a subsolution of (3.1) for every nonpositive constant $C \leq 0$. This subsolution is suitable in the case (i).

In the case (ii), we need to build a subsolution which satisfies (3.24), which is more tricky. Note that $F$ and $f$ given by (3.22) still satisfy (3.3)(3.5). Since the compatibility condition (3.23) holds, we can use the result of [13, Prop. 4.7]: there exists a subsolution $\psi$ of

$$
F(x, D \psi)=f(x), \quad x \in \mathbb{T}^{N} \quad \text { with } \quad \psi(x)=\frac{g(x)}{\sum_{i=1}^{m} \Lambda_{i}(x)} \quad \text { on } \mathcal{F} .
$$

Then, the following computation shows that $\underline{\Psi}:=(\psi, \ldots, \psi)$ is a subsolution of (3.1) such that (3.24) holds: recalling (2.3), for all $x \in \mathbb{T}^{N}$,

$F_{i}\left(x, D \underline{\Psi}_{i}\right)+\sum_{j=1}^{m} d_{i j} \underline{\Psi}_{j}=F_{i}(x, D \psi)+\psi(x) \sum_{j=1}^{m} d_{i j} \leq F(x, D \psi) \leq f(x) \leq f_{i}(x)$.

Now we turn to the construction of a supersolution. Under assumption (3.8), we can use $\psi_{C}$ again with a suitable choice of $C \geq 0$ since, for all $i$,

$$
F_{i}\left(x, D\left(\psi_{C}\right)_{i}(x)\right)+\sum_{j=1}^{m} d_{i j}(x)\left(\psi_{C}\right)_{i}(x)=F_{i}(x, 0)+\left(\sum_{j=1}^{m} d_{i j}(x)\right) C \geq \eta C
$$

where

$$
\eta=\inf _{\mathbb{T}^{N}} \sum_{j=1}^{m} d_{i j}>0
$$

by (3.8). It then suffices to choose

$$
C \geq \eta^{-1} \max _{1 \leq i \leq m}\left|f_{i}\right|_{\infty}
$$

In the second case, since $\mathcal{F} \neq \emptyset$, we may define $d_{\mathcal{F}}(x)=\operatorname{dist}(x, \mathcal{F})$. Choose $C$ as in (3.25) with $\eta=1$. By coercivity of $F_{i}$ (see (3.4)), there exists $C^{\prime}>0$ such that, for all $x \in \mathbb{T}^{N}, p \in \mathbb{R}^{N}$, if $|p| \geq C^{\prime}$, then $F_{i}(x, p) \geq C$ for all $i$. We claim that $\bar{\Psi}=(\bar{\psi}, \ldots, \bar{\psi})$ with $\bar{\psi}=C^{\prime} d_{\mathcal{F}}+C$, is a viscosity supersolution of the stationary problem. Indeed, let $i \in\{1, \ldots, m\}$ and let $\varphi$ be a $C^{1}$ function such that $\bar{\psi}-\varphi$ achieves a minimum at $x_{0} \notin \mathcal{F}$. In $\mathbb{T}^{N} \backslash \mathcal{F}$, it is well-known that $\left|D d_{\mathcal{F}}\right|=1$ in the viscosity sense. Since $d_{\mathcal{F}}+C / C^{\prime}-\varphi / C^{\prime}$ achieves a minimum at $x_{0}$, we get $\left|D \varphi\left(x_{0}\right)\right| \geq C^{\prime}$. It follows that

$$
F_{i}\left(x_{0}, D \varphi\left(x_{0}\right)\right)+\left(\sum_{j=1}^{m} d_{i j}\left(x_{0}\right)\right) \bar{\psi}\left(x_{0}\right) \geq C \geq f_{i}\left(x_{0}\right)
$$

since both $\sum_{j=1}^{m} d_{i j}\left(x_{0}\right)$ and $\bar{\psi}\left(x_{0}\right)$ are nonnegative. If $x_{0} \in \mathcal{F}$, then $f_{i}\left(x_{0}\right)$ vanishes. Since $F_{i} \geq 0$, the supersolution inequality obviously holds on $\mathcal{F}$. The claim is proved. 
Then, we apply the extension of Perron's method to systems, see $[10,16]$. Using the comparison principle 3.3 and following readily the proof of [10, Proposition 2.1], we obtain that the supremum of subsolutions which are less than $\psi_{C}$ (resp. $\left.\bar{\Psi}\right)$ is a solution in the case (i) (resp. (ii)). From Lemma 3.5, the subsolutions of (3.1) are Lipschitz continuous with a constant $L$ depending only on $H_{1}, \ldots, H_{m}$ and $D$. It follows that the supremum is still Lipschitz continuous. In the case of (ii), note that the supremum still satisfies (3.24).

Remark 3.8. Under the assumptions (2.1), (3.3)-(3.5) there always exists a subsolution. The assumption $\mathcal{F} \neq \emptyset$ is needed to build a supersolution when for instance $\mathcal{D}_{i}=\mathbb{T}^{N}$ for all $i$. Notice that, in the case (i) of Proposition 3.6, we do not need to assume (3.5), the $f_{i}$ 's may be any continuous functions in $\mathbb{T}^{N}$.

\section{The ergodic problem}

In this section, we study the solutions of (3.1) with an ergodic constant, that is:

$$
H_{i}\left(x, D v_{i}\right)+\sum_{j=1}^{m} d_{i j}(x) v_{j}=c_{i} \quad x \in \mathbb{T}^{N}, 1 \leq i \leq m,
$$

where $H_{i}$ is given by (3.2). When (3.8) holds, then, see Remark 3.8, for any $c=\left(c_{1}, \ldots, c_{m}\right)$ there is a unique viscosity solution $v \in C\left(\mathbb{T}^{N}\right)$. Hence we concentrate on the case $\mathcal{D}_{i}$ not empty for some $i$ and we consider the ergodic approximation to (4.1): for $\lambda \in(0,1)$, let $v^{\lambda}=\left(v_{1}^{\lambda}, \ldots, v_{m}^{\lambda}\right)$ be the solution of

$$
\lambda v_{i}+H_{i}\left(x, D v_{i}\right)+\sum_{j=1}^{m} d_{i j}(x) v_{j}=0 \quad x \in \mathbb{T}^{N}, 1 \leq i \leq m .
$$

Lemma 4.1. Assume (2.1), (2.4) with $E_{2}=\mathbb{T}^{N}$ and (3.3)-(3.5). Then there exist a unique viscosity solution $v^{\lambda}$ of (4.2) and some constants $C_{0}, M>0$ independent of $\lambda$ such that $v^{\lambda}$ is Lipschitz continuous with constant $C_{0}$ and

$$
0 \leq v_{i}^{\lambda} \leq \frac{M}{\lambda} \quad \text { and } \quad\left|\sum_{j=1}^{m} d_{i j} v_{j}^{\lambda}\right| \leq M, \quad i=1, \ldots, m .
$$

Proof. We first observe that the system (4.2) satisfies the assumption (3.8) for all $\lambda>0$ so Theorem 3.3 and Proposition 3.6 (classical case) hold. Hence there exists a unique solution $v^{\lambda}$. Moreover, since $\bar{u}=(M / \lambda, \ldots, M / \lambda), \underline{u}=$ $(-M / \lambda, \ldots,-M / \lambda)$, where

$$
M \geq \sup _{1 \leq i \leq m} \sup _{x \in \mathbb{T}^{N}}\left|F_{i}(x, 0)\right|+\left|f_{i}(x)\right|
$$

are, respectively, a super and a subsolution of (4.2), we have

$$
-\frac{M}{\lambda} \leq v_{i}^{\lambda} \leq \frac{M}{\lambda} \quad i=1, \ldots, m .
$$


In fact, in our case where $H_{i}(x, p)=F_{i}(x, p)-f_{i}(x)$ with $f_{i} \geq 0$ and $F_{i}(x, 0)=0$, we have that $(0, \ldots, 0)$ is a subsolution and therefore we obtain the more precise estimate

$$
0 \leq v_{i}^{\lambda} \quad i=1, \ldots, m
$$

We want to prove that the $v_{i}^{\lambda}$ 's are Lipschitz continuous uniformly with respect to $\lambda$. We argue as in the second part of the proof of Lemma 3.5, using now Lemma 2.6 (ii) with $E_{1}=\emptyset$ and $E_{2}=\mathbb{T}^{N}$, since (2.3) is not assumed here. Equality (3.21) is replaced with

$$
\lambda \sum_{i=1}^{m} \Lambda_{i}(x) v_{i}^{\lambda}+\sum_{i=1}^{m} \Lambda_{i}(x) F_{i}\left(x, D v_{i}^{\lambda}\right)+\sum_{i, j=1}^{m} \Lambda_{i}(x) d_{i j}(x) v_{j}^{\lambda}=\sum_{i=1}^{m} \Lambda_{i}(x) f_{i}(x)
$$

and

$$
\lambda \sum_{i=1}^{m} \Lambda_{i}(x) v_{i}^{\lambda}+\sum_{j=1}^{m}\left(\sum_{i=1}^{m} \Lambda_{i}(x) d_{i j}(x)\right) v_{j}^{\lambda} \geq 0
$$

since $D(x)^{T} \Lambda(x) \geq 0$ and (4.5) holds. We then conclude as in Lemma 3.5 that $v^{\lambda}$ is Lipschitz continuous with some constant $C_{0}=C_{0}\left(H_{1}, \ldots, H_{m}, D\right)$.

Finally, since for all $i, \lambda v_{i}^{\lambda}$ and $H_{i}\left(x, D v_{i}^{\lambda}\right)$ are bounded independently of $\lambda$ in (4.2), it is true also for $\sum_{j=1}^{m} d_{i j} v_{j}^{\lambda}$.

The next theorem gives a first set of assumptions under which we may solve (4.1).

Theorem 4.2. We assume (2.1), (2.4) with $E_{2}=\mathbb{T}^{N},(3.3)-(3.5)$ and

$$
\mathcal{F}=\left\{x \in \mathbb{T}^{N}: \sum_{i=1}^{m} f_{i}(x)=0\right\} \neq \emptyset \text {. }
$$

Let $x^{*} \in \mathcal{F}$. If $v^{\lambda}$ is the solution of (4.2), then, up to extract some subsequence as $\lambda \rightarrow 0$,

$$
\begin{aligned}
& -\lambda v^{\lambda} \rightarrow c=(0, \ldots, 0) \in \mathbb{R}^{m}, \\
& v^{\lambda}-v^{\lambda}\left(x^{*}\right) \rightarrow v=\left(v_{1}, \ldots, v_{m}\right) \text { in } C\left(\mathbb{T}^{N}\right)
\end{aligned}
$$

and $(c, v) \in \mathbb{R}^{m} \times C\left(\mathbb{T}^{N}\right)$ is solution to (4.1) with $v$ Lipschitz continuous. Moreover

$$
v_{i}(x)=0 \quad \text { for all } x \in \mathcal{F}, 1 \leq i \leq m,
$$

and $c=(0, \ldots, 0)$ is the unique constant vector in $\operatorname{ker} D(x)$, for all $x \in \mathbb{T}^{N}$, such that (4.1) has a solution.

Proof. Fix $x^{*} \in \mathbb{T}^{N}$ and let $w^{\lambda}(x)=v^{\lambda}(x)-v^{\lambda}\left(x^{*}\right)$. From Lemma 4.1, $w^{\lambda}$ is Lipschitz continuous and bounded since $\mathbb{T}^{N}$ is bounded. From Ascoli's theorem, up to subsequences, there exist a constant $c \in \mathbb{R}^{m}$ and a Lipschitz continuous function $v$ such that

$$
-\lambda v^{\lambda}\left(x^{*}\right) \rightarrow c \quad \text { and } \quad w^{\lambda} \rightarrow v \quad \text { in } \quad C\left(\mathbb{T}^{N}\right) \quad \text { as } \lambda \rightarrow 0 .
$$


Notice that $v$ depends on $x^{*}$ but not $c$ since, for any $x^{*}, y^{*} \in \mathbb{T}^{N}$,

$$
\left|-\lambda v^{\lambda}\left(x^{*}\right)+\lambda v^{\lambda}\left(y^{*}\right)\right| \leq \lambda C_{0}\left|x^{*}-y^{*}\right| \rightarrow 0 \quad \text { as } \lambda \rightarrow 0 .
$$

Moreover, multiplying (4.2) by $\lambda$ for all $i$ and sending $\lambda \rightarrow 0$, we obtain $-\sum_{j} d_{i j}(x) c_{i}=0$ which gives $D(x) c=0$ and therefore $c \in \operatorname{ker} D(x)$.

Let $x \in \mathcal{F}$. Since $F_{i} \geq 0$ and $f_{i}(x)=0$, we observe that (4.2) implies

$$
\lambda v_{i}^{\lambda}(x)+\sum_{j=1}^{m} d_{i j}(x) v_{j}^{\lambda}(x) \leq 0 \quad i=1, \ldots, m .
$$

By Lemma 2.6 (ii), there exists a continuous $\Lambda: \mathbb{T}^{N} \rightarrow \mathbb{R}^{m}, \Lambda>0$ such that $D(x)^{T} \Lambda(x) \geq 0$ on $\mathbb{T}^{N}$ and, since $v_{i}^{\lambda}(x) \geq 0$ by (4.5), we obtain

$$
\lambda \sum_{i=1}^{m} \Lambda_{i}(x) v_{i}^{\lambda}(x) \leq \lambda \sum_{i=1}^{m} \Lambda_{i}(x) v_{i}^{\lambda}(x)+\sum_{j=1}^{m} v_{j}^{\lambda}(x)\left(\sum_{i=1}^{m} \Lambda_{i}(x) d_{i j}(x)\right) \leq 0 .
$$

It follows

$$
v_{i}^{\lambda}=0 \quad \text { on } \mathcal{F}, \quad i=1, \ldots, m .
$$

From now on, we choose $x^{*} \in \mathcal{F}$. From (4.11), we obtain that $c=$ $(0, \ldots, 0)$ and $v$ vanishes on $\mathcal{F}$. Observe that $w^{\lambda}$ is a solution of the system

$\lambda w_{i}^{\lambda}+H_{i}\left(x, D w_{i}^{\lambda}\right)+\sum_{j=1}^{m} d_{i j}(x) w_{j}^{\lambda}(x)+\sum_{j=1}^{m} d_{i j}(x) v_{j}^{\lambda}\left(x^{*}\right)=0 \quad$ in $\mathbb{T}^{N}, 1 \leq i \leq m$,

and that the last term in the left-hand side is 0 because of (4.11). By (4.8) and the stability for viscosity solutions as $\lambda \rightarrow 0$, we conclude that the couple $((0, \ldots, 0), v)$ is a solution of the system $(4.1)$.

Suppose that $(c, v)$ and $(\tilde{c}, \tilde{v})$ are two solutions of (4.1) with $c, \tilde{c} \in$ ker $D(x)$ for all $x$. Define $w(x, t)=v(x)-c t$ and $\tilde{w}(x, t)=\tilde{v}(x)-\tilde{c} t$. Since $c, \tilde{c} \in \operatorname{ker} D(x)$, we have that $w$ and $\tilde{w}$ are solutions of (5.1) with initial conditions $v$ and $\tilde{v}$ respectively. By comparison (see Proposition 5.1), we get that, for all $x \in \mathbb{T}^{N}, t \geq 0$ and $1 \leq i \leq m$,

$$
v_{i}(x)-\tilde{v}_{i}(x)+\left(\tilde{c}_{i}-c_{i}\right) t \leq \max _{1 \leq j \leq m} \sup _{\mathbb{T}^{N}}\left(v_{j}-\tilde{v}_{j}\right)^{+} .
$$

Therefore $\tilde{c}_{i} \leq c_{i}$. Exchanging the role of $v$ and $\tilde{v}$, we obtain that $c=\tilde{c}$.

We give another case where we can solve the ergodic problem (4.1). In particular, note that $\mathcal{F}$ may be empty.

Theorem 4.3. We assume that

$$
D \text { does not depend on } x
$$

and that (2.1), (2.3), (2.4) and (3.3)-(3.5) hold. Then, there is at least one solution $(c, v) \in \mathbb{R}^{m} \times C\left(\mathbb{T}^{N}\right)$ to (4.1) with $v$ Lipschitz continuous, $c=\left(c_{1}, \ldots, c_{1}\right)$ is unique in $\operatorname{ker} D$ and

$$
\sum_{i=1}^{m} \Lambda_{i} \min _{\mathbb{T}^{N}} f_{i}(x) \leq-c_{1} \sum_{i=1}^{m} \Lambda_{i} \leq \min _{\mathbb{T}^{N}} \sum_{i=1}^{m} \Lambda_{i} f_{i}(x),
$$

where the constant vector $\Lambda>0$ is given by Lemma 2.6 (i). 
Proof. Let $v^{\lambda}$ be the solution of (4.2) and fix any $x^{*} \in \mathbb{T}^{N}$. We argue as in the proof of Theorem 4.2. From Lemma 4.1 and Ascoli's theorem, there exist $c=\left(c_{1}, \ldots, c_{m}\right) \in \mathbb{R}^{m}$ and $v \in C\left(\mathbb{T}^{N}\right)$ such that, up to extract subsequences, for $i=1, \ldots, m$,

$$
\lambda v_{i}^{\lambda}\left(x^{*}\right) \rightarrow-c_{i} \quad \text { and } \quad v_{i}^{\lambda}-v_{i}^{\lambda}\left(x^{*}\right) \rightarrow v_{i} \text { in } C\left(\mathbb{T}^{N}\right), \quad \text { as } \lambda \rightarrow 0 .
$$

Notice that $c_{i}$ does not depend on the choice of $x^{*}$, (see (4.9)), and $c \in \operatorname{ker} D$. We write

$\lambda v_{i}^{\lambda}+H_{i}\left(x, D\left(v_{i}^{\lambda}-v_{i}^{\lambda}\left(x^{*}\right)\right)\right)+\sum_{j=1}^{m} d_{i j}\left(v_{j}^{\lambda}-v_{i}^{\lambda}\left(x^{*}\right)\right)+\sum_{j=1}^{m} d_{i j} v_{j}^{\lambda}\left(x^{*}\right)=0 \quad$ in $\mathbb{T}^{N}$,

From (4.3), we obtain that some subsequences of both $\lambda v_{i}^{\lambda}\left(x^{*}\right)$ and $\sum_{j=1}^{m} d_{i j}$ $v_{j}^{\lambda}\left(x^{*}\right)$ converge. We call the second limit $\rho_{i}=\rho_{i}\left(x^{*}\right)$. Using the positive vector $\Lambda$ given by Lemma 2.6 (i), we have

$$
\sum_{i=1}^{m} \Lambda_{i} \sum_{j=1}^{m} d_{i j} v_{j}^{\lambda}\left(x^{*}\right)=\sum_{j=1}^{m}\left(\sum_{i=1}^{m} \Lambda_{i} d_{i j}\right) v_{j}^{\lambda}\left(x^{*}\right)=0 .
$$

Passing to the limit in the above formula, it follows $\langle\Lambda, \rho\rangle=0$, where $\langle\cdot, \cdot\rangle$ is the standard inner product. Since $D^{T} \Lambda=0$ and the rank of $D$ is $m-1$, we get that the image $\operatorname{im} D$ of $D$ is $\Lambda^{\perp}$. Thus $\rho \in \operatorname{im} D$ and there exists $\tilde{\rho} \in \mathbb{R}^{m}$ such that $D \tilde{\rho}=\rho$. We then use the stability result for viscosity solutions and pass to the limit in (4.14). We get

$$
H_{i}\left(x, D v_{i}\right)+\sum_{j=1}^{m} d_{i j} v_{j}(x)+\sum_{j=1}^{m} d_{i j} \tilde{\rho}_{j}=c_{i} \quad \text { in } \mathbb{T}^{N}, 1 \leq i \leq m .
$$

Then $(c, v(\cdot)+\tilde{\rho})$ is solution to (4.1). The function $\tilde{v}=v+\tilde{\rho}$ depends on $x^{*}$ but not $c$.

From Lemma 2.6, the kernel of $D$ is the line spanned by $(1, \ldots, 1)$. Thus, any $c \in \operatorname{ker} D$ has the form $\left(c_{1}, \ldots, c_{1}\right)$. The proof of uniqueness of $c$ is the same as the one in Theorem 4.2 .

It remains to prove (4.13). We use again the vector $\Lambda$ given by Lemma 2.6 (i). On the one hand, multiplying (4.1) by $\Lambda_{i}$ and summing them for $i=$ $1, \ldots, m$, we obtain

$$
0 \leq \sum_{i=1}^{m} \Lambda_{i} f_{i}(x)+c_{1} \sum_{i=1}^{m} \Lambda_{i}, \quad x \in \mathbb{T}^{N}
$$

On the other hand, let $x_{i} \in \mathbb{T}^{N}$ be a minimum of the continuous function $u_{i}$ and set $\bar{u}_{i}=u_{i}\left(x_{i}\right)$. At $x_{i}$, the equation (4.1) reads

$$
\sum_{j=1}^{m} d_{i j} \bar{u}_{j} \geq \sum_{j=1}^{m} d_{i j} u_{j}\left(x_{i}\right)=f_{i}\left(x_{i}\right)+c_{1}
$$


since $\bar{u}_{j} \leq u_{j}\left(x_{i}\right)$ and $d_{i j} \leq 0$ for $i \neq j$. Multiplying (4.17) by $\Lambda_{i}$ and summing them for $i=1, \ldots, m$, we get

$$
0=\sum_{j=1}^{m} \bar{u}_{j} \sum_{i=1}^{m} \Lambda_{i} d_{i j} \geq \sum_{i=1}^{m} \Lambda_{i}\left(f_{i}\left(x_{i}\right)+c_{1}\right) \geq \sum_{i=1}^{m} \Lambda_{i}\left(\min _{\mathbb{T}^{N}} f_{i}+c_{1}\right) .
$$

Combining the previous inequality with (4.16), we finally obtain (4.13).

\section{Remark 4.4.}

(1) The inequality (4.13) gives a characterization of $c$ when all the $f_{i}$ 's achieve the same minimum at the same point.

(2) It would be interesting to prove Theorem 4.3 when the $d_{i j}$ 's depend on $x$. The difficulty is that the $\rho_{i}$ 's in the proof are now functions on $x$ and we do not obtain anymore a solution of (4.1).

Example 4.5. Consider the system

$$
\left\{\begin{array}{l}
\left|D v^{1}\right|+v^{1}-v^{2}=f_{1}+c_{1} \quad \text { in } \mathbb{T}^{N}, \\
\left|D v^{2}\right|-v^{1}+v^{2}=f_{2}+c_{2} \quad \text { in } \mathbb{T}^{N},
\end{array}\right.
$$

with $f_{1} \equiv a, f_{2} \equiv b, a, b>0$. This system satisfies all the assumptions of Theorem 4.2 with the exception of (4.6) since $f_{1}+f_{2} \equiv a+b>0$. However, all the assumptions of Theorem 4.3 hold and therefore we can solve (4.18). For instance, we can exhibit constant solutions $(c, v)$ with $c_{1}=c_{2}$. An easy computation gives $c_{1}=-(a+b) / 2$ and all constants $v=\left(v_{1}, v_{2}\right)$ satisfying $v_{1}-v_{2}=(a-b) / 2$ are suitable. Notice that we can solve (4.18) even in some cases where Theorem 3.3 and Proposition 3.6 do not apply. Indeed, (4.18) corresponds to the degenerate case of Theorem 3.3 and either $f_{1}+c_{1}$ or $f_{2}+c_{1}$ is negative and (3.5) does not hold.

\section{Large time behavior}

We are interested in the long-time behavior of the evolutive system

$$
\left\{\begin{array}{l}
\frac{\partial u_{i}}{\partial t}+H_{i}\left(x, D u_{i}\right)+\sum_{j=1}^{m} d_{i j}(x) u_{j}=0(x, t) \in \mathbb{T}^{N} \times(0,+\infty), \\
u_{i}(x, 0)=u_{0, i}(x) \\
x \in \mathbb{T}^{N}, \quad i=1, \ldots m,
\end{array}\right.
$$

where $H_{i}$ is of the form (3.2) and

$$
u_{0, i} \text { is continuous and 1-periodic. }
$$

We start giving some auxiliary results for the evolutive problem. The following two propositions come from [8], where homogenization of a general class of monotone systems which includes in particular the weakly coupled system (5.1), is studied. Let us mention that Proposition 5.1 is established in [8] under the additional assumption

There exists a modulus of continuity $\omega$ such that,

$$
\left|F_{i}(x, p)-F_{i}(y, p)\right| \leq \omega((1+|p|)|x-y|) \quad \text { for all } x, y \in \mathbb{T}^{N}, p \in \mathbb{R}^{N}, \quad i=1, \ldots, m,
$$


but a careful examination of the proof shows that we do not need it. The coercivity of the $F_{i}$ 's is enough (see the proof of Theorem 3.3).

Proposition 5.1. Assume (2.1) and (3.2)-(3.5).

(i) If $u_{0}, v_{0}$ are two initial datas satisfying (5.2) and $u, v$ are respectively $a$ viscosity subsolution and a supersolution of (5.1), then for any $t \geq 0$,

$$
\max _{1 \leq i \leq m} \sup _{\mathbb{T}^{N}}\left(u_{i}(\cdot, t)-v_{i}(\cdot, t)\right) \leq \max _{1 \leq i \leq m} \sup _{\mathbb{T}^{N}}\left(u_{i}(\cdot, 0)-v_{i}(\cdot, 0)\right)^{+} .
$$

(ii) For any $u_{0}$ satisfying (5.2), there exists a unique continuous viscosity solution $u$ of (5.1).

A crucial step in the study of the large-time behavior of equations or systems is to obtain compactness properties of the sequence $(u(\cdot, t))_{t \geq 0}$. As for a single Hamilton-Jacobi equation, it relies on the coercitivity of the Hamiltonians.

Proposition 5.2. Under the assumptions of Theorem 4.2 or Theorem 4.3, let $u_{0} \in W^{1, \infty}\left(\mathbb{T}^{N}\right)$ and $u$ be the solution of (5.1) with initial datum $u_{0}$. Then

$$
\begin{array}{ll}
|u(x, t)+c t| \leq C & x \in \mathbb{T}^{N}, t \in[0, \infty), \\
|u(x, t)-u(y, s)| \leq L(|x-y|+|t-s|) & x, y \in \mathbb{T}^{N}, t, s \in[0, \infty),
\end{array}
$$

with $C, L$ independent of time, where $c$ is the ergodic constant given in Theorem 4.2 or 4.3 . It follows that

$$
\frac{u_{i}(x, t)}{t} \rightarrow-c_{i} \quad \text { uniformly in } \mathbb{T}^{N} \text { as } t \rightarrow+\infty, \quad i=1, \ldots, m .
$$

Remark 5.3. Given $u_{0}$ satisfying (5.2), set $S(t) u_{0}=u(x, t)$ for $t \geq 0$ where $u$ is the solution of (5.1) with initial datum $u_{0}$. Then it is easy to see that $S(\cdot)$ generates a nonlinear, monotone, nonexpansive semigroup in $C\left(\mathbb{T}^{N} ; \mathbb{R}^{m}\right)$. Under the assumptions of Theorem $4.2, c=(0, \ldots, 0)$, so $u$ is in $L^{\infty}\left(\mathbb{T}^{N} \times[0,+\infty)\right)$. It follows that $\left\{S(t) u_{0}, t \geq 0\right\}$ is relatively compact in $C\left(\mathbb{T}^{N}\right)$. Therefore, the $\omega$-limit set of an initial datum $u_{0}$ with respect to the semigroup $S(t)$,

$\omega\left(u_{0}\right)=\left\{\psi=\left(\psi_{1}, \ldots, \psi_{m}\right) \in C\left(\mathbb{T}^{N}\right): \exists t_{n} \rightarrow \infty\right.$ such that $\left.\lim _{n \rightarrow \infty} S\left(t_{n}\right) u_{0}=\psi\right\}$.

is nonempty.

Proof. The proof is based on the existence of a solution to the ergodic problem which is used to estimate $u$. It is classical but we provide the proof for reader's convenience.

Let $(c, v)$ be the solution of (4.1) given by Theorem 4.2 or 4.3. Since $c \in$ $\operatorname{ker} D, w(x, t)=v(x)-c t-\left|u_{0}\right|_{\infty}-|v|_{\infty}$ and $\tilde{w}(x, t)=v(x)-c t+\left|u_{0}\right|_{\infty}+|v|_{\infty}$ are respectively viscosity subsolution and supersolution to (5.1). By comparison (5.3), it follows that

$$
v(x)-\left|u_{0}\right|_{\infty}-|v|_{\infty} \leq u(x, t)+c t \leq v(x)+\left|u_{0}\right|_{\infty}+|v|_{\infty} \quad(x, t) \in \mathbb{T}^{N} \times[0, \infty),
$$


which proves (5.4). If we define

$C:=\sup \left\{\left|H_{i}(x, p)+\sum_{j} d_{i j} r_{j}\right|: x \in \mathbb{R}^{N},|r| \leq\left|u_{0}\right|_{\infty},|p| \leq\left|D u_{0}\right|_{\infty}, 1 \leq i \leq m\right\}$,

it is easy to see that $v^{ \pm}(x, t)=\left(u_{0,1}(x) \pm C t, \ldots, u_{0, m}(x) \pm C t\right)$ are viscosity subsolution and supersolution of (5.1). By Proposition 5.1, it follows

$$
v^{-} \leq u \leq v^{+} \quad \text { in } \mathbb{T}^{N} \times[0,+\infty)
$$

Let $h \geq 0$ and note that, since the $H_{i}$ 's are independent of $t, u(\cdot, \cdot+h)$ is still a solution of (5.1) with initial data $u(\cdot, h)$. By (5.3) and (5.8), we get for all $i=1, \ldots, m,(x, t) \in \mathbb{R}^{N} \times[0,+\infty)$,

$$
u_{i}(x, t+h)-u_{i}(x, t) \leq \max _{1 \leq j \leq m} \sup _{\mathbb{T}^{N}}\left(u_{j}(\cdot, h)-u_{0, j}\right)^{+} \leq C h,
$$

and therefore $u_{i}$ is Lipschitz continuous with respect to $t$ for every $x$ with

$$
\left|\frac{\partial u_{i}}{\partial t}\right|_{\infty} \leq C
$$

with $C$ is independent of $t$. From (5.1) and (5.7), we obtain, in the viscosity sense,

$$
F_{i}\left(x, D u_{i}\right) \leq C^{\prime} \quad(x, t) \in \mathbb{T}^{N} \times[0,+\infty) .
$$

It follows from Lemma 3.5 that $u_{i}$ is Lipschitz continuous in $x$ for every $t$ with $\left|D u_{i}\right|_{\infty} \leq L_{i}$ (with $L_{i}$ independent of $\left.t\right)$.

We now state and prove our convergence result in the case of systems, under the assumptions of Theorem 4.2.

Theorem 5.4. Assume (2.1), (2.4) with $E_{2}=\mathbb{T}^{N}$, and (3.3)-(3.5). Suppose that $\mathcal{A} \neq \emptyset$. For every $u_{0}$ satisfying (5.2), there exists a solution $u_{\infty}$ to (3.1) such that the solution $u$ of (5.1) with initial datum $u_{0}$ satisfies

$$
\lim _{t \rightarrow \infty}\left|u(\cdot, t)-u_{\infty}\right|_{\infty}=0 .
$$

Moreover $\left(u_{\infty}\right)_{i}=\left(u_{\infty}\right)_{j}$ on $\mathcal{A}$ for all $i, j$.

Proof. The proof is divided in several steps.

Step 1. Reduction to Lipschitz initial datas. Given $u_{0}$ satisfying (5.2), set $S(t) u_{0}=u(x, t)$. Since the semigroup $S(t)$ is nonexpansive, see Remark 5.3, it is sufficient to show the result for $u_{0} \in W^{1, \infty}\left(\mathbb{T}^{N}\right)$.

Step 2. A positive linear combination of the $u_{i}$ 's is nonincreasing on $\mathcal{A}$. Since (2.3) holds with $E_{1}=\mathcal{A}$, from (2.4) and Lemma 2.6, there exists a positive continuous function $\Lambda=\left(\Lambda_{1}, \ldots, \Lambda_{m}\right): \mathcal{A} \rightarrow \mathbb{R}^{m}$ such that $D(x)^{T} \Lambda(x)=0$ on $\mathcal{A}$. By multiplying the equations (5.1) by $\Lambda_{i}>0$ and summing for $i=1, \ldots, m$, we obtain 


$$
\begin{gathered}
\frac{\partial}{\partial t}\left(\sum_{i=1}^{m} \Lambda_{i} u_{i}\right)+\sum_{i=1}^{m} \Lambda_{i} F_{i}\left(x, D u_{i}\right)+\sum_{j=1}^{m}\left(\sum_{i=1}^{m} \Lambda_{i} d_{i j}\right) u_{j} \\
=\sum_{i=1}^{m} \Lambda_{i} f_{i} \quad \text { in } \mathcal{A} \times(0,+\infty),
\end{gathered}
$$

in the viscosity sense. Formally, since $F_{i} \geq 0, D(x)^{T} \Lambda(x)=0$ and $f_{i}(x)=0$ for $x \in \mathcal{A}$, it follows that

$$
\frac{\partial}{\partial t}\left(\sum_{i=1}^{m} \Lambda_{i} u_{i}\right) \leq 0 \quad \text { in } \mathcal{A} \times(0,+\infty)
$$

and we obtain

Lemma 5.5. There exists a Lipschitz continuous $\phi: \mathcal{A} \rightarrow \mathbb{R}$ such that

$$
\sum_{i=1}^{m} \Lambda_{i} u_{i}(\cdot, t) \downarrow \phi \quad \text { uniformly on } \mathcal{A} \text { as } t \rightarrow+\infty \text {. }
$$

The details are in the proof of the lemma which is postponed.

Step 3. Uniform convergence of a subsequence of $u$. Notice that the assumptions of Theorem 4.2 hold. It follows that there exists a solution to the ergodic problem (4.1) and therefore, from Proposition 5.2 (see also Remark 5.3), $(u(\cdot, t))_{t \geq 0}$ is relatively compact in $C\left(\mathbb{T}^{N}\right)$ and there exists a sequence $t_{n} \rightarrow$ $+\infty$ such that $u\left(\cdot, t_{n}\right)$ converges uniformly on $\mathbb{T}^{N}$ as $n \rightarrow+\infty$. From (5.3), we obtain that for all $n, q \in \mathbb{N}$,

$$
\max _{1 \leq i \leq m} \sup _{\mathbb{T}^{N} \times[0,+\infty)}\left|u_{i}\left(\cdot, t_{n}+\cdot\right)-u_{i}\left(\cdot, t_{q}+\cdot\right)\right| \leq \max _{1 \leq i \leq m} \sup _{\mathbb{T}^{N}}\left|u_{i}\left(\cdot, t_{n}\right)-u_{i}\left(\cdot, t_{q}\right)\right|
$$

and therefore $\left(u\left(\cdot, t_{n}+\cdot\right)\right)_{n}$ is a Cauchy sequence in $W^{1, \infty}\left(\mathbb{T}^{N} \times[0,+\infty)\right)$. Thus it converges uniformly to some function $w \in W^{1, \infty}\left(\mathbb{T}^{N} \times[0,+\infty)\right)$. By the stability of viscosity solutions, $w$ is a viscosity solution of $(5.1)$ (see $[1,2,10]$ for details).

Step 4. Uniform convergence of the sequence $w(\cdot, t)$ on $\mathcal{A}$. The reason of introducing $w$ in Step 3 is that, from Lemma 5.5, we have

$$
\sum_{i=1}^{m} \Lambda_{i} w_{i}(\cdot, t)=\phi \quad \text { on } \mathcal{A} \text { for all } t \in[0,+\infty) .
$$

Surprisingly, this is enough to prove the convergence of each $w_{i}$ on $\mathcal{A}$ :

Lemma 5.6. The function $w(\cdot, t)$ converges uniformly on $\mathcal{A}$ to a function $u_{\infty}(\cdot)$ which is Lipschitz continuous on $\mathcal{A}$.

The complete proof is postponed, we only outline the main ideas here. At first, from (5.11), we get

$$
\frac{\partial}{\partial t}\left(\sum_{i=1}^{m} \Lambda_{i} w_{i}\right)=0 \quad \text { in } \mathcal{A} \times(0,+\infty) .
$$


Then, writing (5.9) for $w$ and using in addition $D(x)^{T} \Lambda(x)=0$ and $f_{i}=0$ on $\mathcal{A}$ we obtain

$$
\sum_{i=1}^{m} \Lambda_{i} F_{i}\left(x, D w_{i}\right)=0 \quad \text { in } \mathcal{A} \times(0,+\infty) .
$$

Thus, for all $i, F_{i}\left(x, D w_{i}\right)=0$ on $\mathcal{A} \times(0,+\infty)$. It follows that, for fixed $x \in \mathcal{A}$, the system (5.1) reduces to a linear differential system in $\mathbb{R}^{m}$,

$$
\frac{\partial w}{\partial t}(x, t)+D(x) w(x, t)=0 \quad t \in[0,+\infty) .
$$

The solution is given by

$$
w(x, t)=\exp (-t D(x)) w(x, 0) .
$$

Since $D$ is an irreducible $M$-matrix, it has 0 as a simple eigenvalue and all the other eigenvalues have positive real part. It follows that both $\exp (-t D(x))$ and $w(x, t)$ converge as $t \rightarrow+\infty$.

Step 5. Convergence of the whole sequence $(w(\cdot, t))_{t \geq 0}$ on $\mathbb{T}^{N}$. Since $w$ is bounded in $\mathbb{T}^{N} \times[0,+\infty)$, we can introduce the relaxed half-limits

$$
\begin{aligned}
& \bar{w}(x)=\left(\limsup _{t \rightarrow+\infty}^{*} w\right)(x)=\lim _{t \rightarrow+\infty} \sup \{w(y, s): y \in B(x, 1 / t), s \geq t\}, \\
& \underline{w}(x)=\left(\liminf _{t \rightarrow+\infty} * w\right)(x)=\lim _{t \rightarrow+\infty} \inf \{w(y, s): y \in B(x, 1 / t), s \geq t\},
\end{aligned}
$$

where the half-limits are taken componentwise. By the stability of viscosity solutions, $\bar{w}$ and $\underline{w}$ are respectively a sub and a supersolution of (4.1) with $c=0$. From Step 4 , we have

$$
\bar{w}_{i}=\underline{w}_{i}=\left(u_{\infty}\right)_{i} \quad \text { on } \mathcal{A} \text { for all } i=1, \ldots, m .
$$

It follows that

$$
\sum_{i=1}^{m} \Lambda_{i} \bar{w}_{i}=\sum_{i=1}^{m} \Lambda_{i} \underline{w}_{i}=\phi \quad x \in \mathcal{A} .
$$

Applying Theorem 3.3 (degenerate case), thanks to (5.13), we obtain $\bar{w} \leq \underline{w}$ in $\mathbb{T}^{N}$ and therefore $\bar{w}=\underline{w}=: u_{\infty}$ is the unique continuous viscosity solution of (3.1) such that $\sum_{i=1}^{m} \Lambda_{i}\left(u_{\infty}\right)_{i}=\phi$ on $\mathcal{A}$. This gives the convergence of $w(\cdot, t)$ to $u_{\infty}$ in $C\left(\mathbb{T}^{N}\right)$ and this latter function is Lipschitz continuous.

Step 6. Convergence of $(u(\cdot, t))_{t \geq 0}$. It remains to prove that $u(\cdot, t)$ converges to $u_{\infty}$ as $t \rightarrow+\infty$. We proceed as in [4]. Since $u$ and $w$ are both solutions of (5.1), from Proposition 5.1, we have

$$
\max _{1 \leq i \leq m} \sup _{\mathbb{T}^{N}}\left|u_{i}\left(\cdot, t_{n}+t\right)-w_{i}(\cdot, t)\right| \leq \max _{1 \leq i \leq m} \sup _{\mathbb{T}^{N}}\left|u_{i}\left(\cdot, t_{n}\right)-w_{i}(\cdot, 0)\right|=o_{n}(1),
$$

where $o_{n}(1) \rightarrow 0$ as $n \rightarrow+\infty$ since $u\left(\cdot, t_{n}+\cdot\right)$ converges uniformly to $w(\cdot, \cdot)$. It follows that, for all $1 \leq i \leq m, x \in \mathbb{T}^{N}$ and $t \geq 0$,

$$
w(x, t)-o_{n}(1) \leq u\left(x, t_{n}+t\right) \leq w(x, t)+o_{n}(1) .
$$

Taking the relaxed half-limits in the above inequality, we obtain

$$
u_{\infty}(x)-o_{n}(1)=\left(\liminf _{t \rightarrow+\infty} w\right)(x)-o_{n}(1) \leq\left(\liminf _{t \rightarrow+\infty} * u\right)(x)
$$


and

$$
\left(\limsup _{t \rightarrow+\infty} * u\right)(x) \leq\left(\limsup _{t \rightarrow+\infty} * w\right)(x)+o_{n}(1)=u_{\infty}(x)+o_{n}(1) .
$$

Sending $n$ to $+\infty$ implies

$$
\left(\liminf _{t \rightarrow+\infty} *\right)(x)=\left(\limsup _{t \rightarrow+\infty} * u\right)(x)=u_{\infty}(x)
$$

which proves the uniform convergence of $u(\cdot, t)$ to $u_{\infty}$ as $t \rightarrow+\infty$.

Step 7. All the $\left(u_{\infty}\right)_{i}$ 's are equal on $\mathcal{A}$. Using the previous arguments, it is straightforward to see that $D(x) u_{\infty}(x) \leq 0$ for all $x \in \mathcal{A}$. From (2.3), it follows that $u_{\infty}(x) \in \operatorname{ker} D(x)$ and therefore, from Lemma 2.6, $\left(u_{\infty}\right)_{i}=\left(u_{\infty}\right)_{j}$ on $\mathcal{A}$ for all $i, j$.

\section{Remark 5.7.}

(1) Let us mention that there is an easy version of the convergence theorem when (2.1), (3.3)-(3.5) and (3.8) holds. In this case, (4.1) has a unique Lipschitz continuous solution $u$ with $c=0$. The solution of (5.1) is bounded and we define the relaxed half-limits as in (5.12). The classical case of Theorem 3.3 yields $\bar{u}=\underline{u}=: u_{\infty}$ and gives the convergence. Actually, in this case, $u_{\infty}=0$.

(2) Assuming $\mathcal{A} \neq \emptyset$, means that all the $f_{i}$ 's are zero at least at a common point (see (3.7) and Remark 3.1). If (2.3) holds with $E_{1}=\mathbb{T}^{N}$ (i.e., if $\cap_{i} \mathcal{D}_{i}=\mathbb{T}^{N}$ in $\left.(3.7)\right)$, then we can replace this assumption with

$$
\exists \bar{f} \geq 0 \text { such that } \min _{\mathbb{T}^{N}} f_{i}=\bar{f} \quad \text { and } \bigcap_{1 \leq i \leq m} \operatorname{argmin} f_{i} \neq \emptyset .
$$

Indeed, using (2.3), we recover the framework of Theorem 5.4 by replacing $u_{i}(x, t)$ with $\tilde{u}_{i}(x, t)=u(x, t)-\bar{f} t$ and $f_{i}$ with $\tilde{f}_{i}-\bar{f} \geq 0$. In a future work, we aim at studying the large time behavior of the system (5.1) when $\mathcal{A}=\emptyset$.

(3) With the same kind of arguments as in Lemma 5.5, we can prove that

$$
\max _{1 \leq j \leq m} u_{j}(x, t) \downarrow \tilde{\phi} \quad \text { uniformly on } \mathcal{A} \text { as } t \rightarrow+\infty \text {. }
$$

The rough idea is that, if $u_{i}(x, t)=\max _{1 \leq j \leq m} u_{j}(x, t)$ for $(x, t)$, then

$$
\sum_{j=1}^{m} d_{i j} u_{j}(x, t) \geq 0
$$

and therefore, using the $i$ th equation, we get $\frac{\partial u_{i}}{\partial t}(x, t) \leq 0$ on $\mathcal{A}$. Note that Lemma 5.5 and (5.15) give an alternative proof for the convergence theorem 5.4 when $m=2$ since these two convergences are enough to imply the convergence of $u_{1}$ and $u_{2}$ on $\mathcal{A}$. This approach is used in $\mathrm{H}$. Mitake and H.V. Tran (In preparation) to obtain the convergence result for two equations in a very similar setting. 
(4) Formula (5.11) means that every element $\psi$ of the $\omega$-limit set $\omega\left(u_{0}\right)$ given by (5.6) satisfies

$$
\sum_{i=1}^{m} \Lambda_{i} \psi_{i}=\phi \quad \text { on } \mathcal{A} \text {. }
$$

Moreover, since $\omega\left(u_{0}\right)$ is positively invariant for the semigroup, for every $\psi \in \omega\left(u_{0}\right)$ the restriction of $S(t) \psi$ to $\mathcal{F}$ is constant in time.

Example 5.8. Extensions to Theorem 5.4 are not easy to obtain. When the assumptions of the previous theorem are not satisfied, the convergence is not always true. The following example is similar to the one in [4]. Consider

$$
\left\{\begin{array}{l}
\frac{\partial u_{1}}{\partial t}+\left|D u_{1}+\alpha\right|+u_{1}-u_{2}=\alpha \text { in } \mathbb{R} \times(0, \infty), \\
\frac{\partial u_{2}}{\partial t}+\left|D u_{2}+\beta\right|-u_{1}+u_{2}=\beta \text { in } \mathbb{R} \times(0, \infty), \\
u_{1}(x, 0)=u_{2}(x, 0)=\sin (x) .
\end{array}\right.
$$

If $\alpha, \beta>1$, then the unique solution of $(5.17)$ is $u(x, t)=(\sin (x-t), \sin (x-t))$ which clearly does not converge as $t \rightarrow \infty$. In this case, (3.3), (3.5), (2.3) and (2.4) hold but (3.4) fails and $\mathcal{A}=\emptyset$. Moreover, notice that the assumptions of Theorem 4.3 hold. The ergodic problem has a solution $(c, v)$ with $c=(0,0)$ and $v=(C, C)$ where $C$ is any constant.

Proof of Lemma 5.5. The proof is inspired from the corresponding one for a scalar equation in [24]. We set

$$
U=\sum_{i=1}^{m} \Lambda_{i} u_{i} \quad \text { and } \quad \Phi=\sum_{i=1}^{m} \Lambda_{i} f_{i}-\sum_{j=1}^{m}\left(\sum_{i=1}^{m} \Lambda_{i} d_{i j}\right) u_{j}
$$

Note that $U$ is Lipschitz continuous with respect to $t$ because of Proposition 5.2 and $\Lambda$ is independent of $t$. Let $\omega(\cdot)$ be a modulus of continuity for $\Phi(\cdot, t)$ on the compact set $\mathbb{T}^{N}$. This modulus is independent of $t$ because of (5.5). Let $x \in \mathcal{A}$ and consider the cube $B_{\varepsilon}(x)=[x-\varepsilon, x+\varepsilon]^{N}$ for $\varepsilon>0$. Noticing that, for all $t \geq 0, \Phi(x, t)=0$ by the very definition of $\mathcal{A}$ and $\Lambda$, we have

$$
\begin{aligned}
\frac{1}{\varepsilon^{n}} \int_{B_{\varepsilon}(x)}[U(y, t+h)-U(y, t)] d y & =\frac{1}{\varepsilon^{n}} \int_{B_{\varepsilon}(x) \times[t, t+h]} \frac{\partial U}{\partial t}(s, y) d s d y \\
& \leq \frac{1}{\varepsilon^{n}} \int_{B_{\varepsilon}(x) \times[t, t+h]} \Phi(y, s) d s d y \\
& \leq h \omega(\varepsilon) .
\end{aligned}
$$

Sending $\varepsilon \rightarrow 0$ and using the continuity of $U$, we get $U(x, t+h) \leq U(x, t)$ for any $h>0$.

Proof of Lemma 5.6. Arguing as in Step 2 of the proof of Theorem 5.4, since $w$ is solution to (5.1), we have 


$$
\frac{\partial}{\partial t}\left(\sum_{i=1}^{m} \Lambda_{i} w_{i}\right)+\sum_{i=1}^{m} \Lambda_{i} F_{i}\left(x, D w_{i}\right)+\sum_{j=1}^{m}\left(\sum_{i=1}^{m} \Lambda_{i} d_{i j}\right) w_{j}=\sum_{i=1}^{m} \Lambda_{i} f_{i}
$$

almost everywhere in $\mathbb{T}^{N} \times(0,+\infty)$.

From now on, we fix $\bar{x} \in \mathcal{A}$. Setting

$W=\sum_{i=1}^{m} \Lambda_{i} w_{i}, \quad \Psi=\sum_{i=1}^{m} \Lambda_{i} F_{i}\left(y, D w_{i}\right) \quad$ and $\quad \Phi=\sum_{i=1}^{m} \Lambda_{i} f_{i}-\sum_{j=1}^{m}\left(\sum_{i=1}^{m} \Lambda_{i} d_{i j}\right) w_{j}$

and integrating as in the proof of Lemma 5.5, we have

$$
\begin{aligned}
& \frac{1}{\varepsilon^{n}} \int_{B_{\varepsilon}(\bar{x})}[W(y, t+h)-W(y, t)] d y \\
& \quad=\frac{1}{\varepsilon^{n}} \int_{B_{\varepsilon}(\bar{x}) \times[t, t+h]} \frac{\partial W}{\partial t}(y, s) d s d y \\
& \quad=\frac{1}{\varepsilon^{n}} \int_{B_{\varepsilon}(\bar{x}) \times[t, t+h]} \Phi(y, s) d s d y-\frac{1}{\varepsilon^{n}} \int_{B_{\varepsilon}(\bar{x}) \times[t, t+h]} \Psi(y, s) d s d y .
\end{aligned}
$$

As in Lemma 5.5 , since $\bar{x} \in \mathcal{A}$,

$$
\frac{1}{\varepsilon^{n}} \int_{B_{\varepsilon}(\bar{x}) \times[t, t+h]} \Phi(y, s) d s d y=o_{\varepsilon}(1),
$$

where $o_{\varepsilon}(1) \rightarrow 0$ as $\varepsilon \rightarrow 0$ uniformly with respect to $t$. Since $W$ does not depend on $t$ on $\mathcal{A}$ from (5.11), we have, for all $y \in B_{\varepsilon}(\bar{x})$,

$$
|W(y, t+h)-W(y, t)| \leq|W(y, t+h)-W(\bar{x}, t+h)|+|W(\bar{x}, t)-W(y, t)|=o_{\varepsilon}(1),
$$

using the uniform continuity of the $d_{i j}$ 's on $\mathbb{T}^{N}$ and the Lipschitz continuity of the $w_{i}$ 's with respect to $y \in \mathbb{T}^{N}$ uniformly in $t$. It follows from (5.18) that

$$
\frac{1}{\varepsilon^{n}} \int_{B_{\varepsilon}(\bar{x}) \times[t, t+h]} \Psi(y, s) d s d y=o_{\varepsilon}(1)
$$

and therefore, for all $1 \leq i \leq m$, using the uniform continuity of $\Lambda_{i}$ on $\mathbb{T}^{N}$ and the boundedness of $F_{i}\left(y, D w_{i}\right)$, we have

$$
\frac{1}{\varepsilon^{n}} \int_{B_{\varepsilon}(\bar{x}) \times[t, t+h]} F_{i}\left(y, D w_{i}(y, s)\right) d s d y=o_{\varepsilon}(1) .
$$

Let $1 \leq i \leq m$ and consider the $i$ th equation:

$$
\frac{\partial w_{i}}{\partial t}+F_{i}\left(y, D w_{i}\right)+\sum_{j=1}^{m} d_{i j} w_{j}=f_{i} \text { almost everywhere in } \mathbb{T}^{N} \times(0,+\infty)
$$


Integrating as above around $\bar{x} \in \mathcal{A}$, we get

$$
\begin{aligned}
& \frac{1}{\varepsilon^{n}} \int_{B_{\varepsilon}(\bar{x})}\left(w_{i}(y, t+h)-w_{i}(y, t)\right) d y+\frac{1}{\varepsilon^{n}} \int_{B_{\varepsilon}(\bar{x}) \times[t, t+h]} \sum_{j=1}^{m} d_{i j} w_{j} d s d y \\
& =\frac{1}{\varepsilon^{n}} \int_{B_{\varepsilon}(\bar{x}) \times[t, t+h]} \frac{\partial w_{i}}{\partial t} d s d y+\frac{1}{\varepsilon^{n}} \int_{B_{\varepsilon}(\bar{x}) \times[t, t+h]} \sum_{j=1}^{m} d_{i j} w_{j} d s d y \\
& =\frac{1}{\varepsilon^{n}} \int_{B_{\varepsilon}(\bar{x}) \times[t, t+h]} f_{i}(y) d s d y-\frac{1}{\varepsilon^{n}} \int_{B_{\varepsilon}(\bar{x}) \times[t, t+h]} F_{i}\left(y, D w_{i}(y, s)\right) d s d y \\
& =o_{\varepsilon}(1)
\end{aligned}
$$

from (5.19) and since $\bar{x} \in \mathcal{A}$. Using the continuity of $w_{i}$ and $d_{i j}$ and sending $\varepsilon$ to 0 , we get, for all $t, h>0$,

$$
w_{i}(\bar{x}, t+h)-w_{i}(\bar{x}, t)+\int_{t}^{t+h} \sum_{j=1}^{m} d_{i j}(\bar{x}) w_{j}(\bar{x}, s) d s=0, \quad 1 \leq i \leq m .
$$

Recalling that $t \mapsto w(\bar{x}, t)$ is Lipschitz continuous, we obtain that $w$ is solution to the linear differential system

$$
\frac{\partial w}{\partial t}(\bar{x}, t)+D(\bar{x}) w(\bar{x}, t)=0 \quad \text { almost everywhere for } t \in(0,+\infty) .
$$

The unique solution of this system is

$$
w(\bar{x}, t)=\exp (-t D(\bar{x})) w(\bar{x}, 0) .
$$

Since $D(\bar{x})$ is an irreducible $M$-matrix, 0 is a simple eigenvalue and all the nonzero eigenvalues have a positive real part. It follows that there exists a matrix $A(\bar{x})$ such that $\exp (-t D(\bar{x}))=A(\bar{x})+O\left(\mathrm{e}^{-r t}\right)$ where $r>0$ is the smallest real part of the nonzero eigenvalues. Therefore,

$$
w(\bar{x}, t) \underset{t \rightarrow+\infty}{\rightarrow} A(\bar{x}) w(\bar{x}, 0)=\lim _{n \rightarrow+\infty} A(\bar{x}) u\left(\bar{x}, t_{n}\right)=: u_{\infty}(\bar{x}) .
$$

Since $w \in W^{1, \infty}\left(\mathbb{T}^{N} \times[0,+\infty)\right)$, we obtain that $u_{\infty} \in W^{1, \infty}\left(\mathbb{T}^{N}\right)$.

\section{The control-theoretic interpretation}

At least when the coefficients $d_{i j}$ of the coupling matrix $D$ are constant, we can give an interpretation of our system of Hamilton-Jacobi equations (5.1) in terms of optimal control of hybrid systems with pathwise deterministic trajectories with random switching. We do not give the proofs here, we refer the readers to Fleming and Zhang [14], Yin and Zhang [27] and the references therein.

Consider the controlled random evolution process $\left(X_{t}, \nu_{t}\right)$ with dynamics

$$
\left\{\begin{array}{l}
\dot{X}_{t}=b_{\nu_{t}}\left(X_{t}, a_{t}\right), \quad t>0 \\
\left(X_{0}, \nu_{0}\right)=(x, i) \in \mathbb{T}^{N} \times\{1, \ldots, m\}
\end{array}\right.
$$


where the control law $a:[0, \infty) \rightarrow A$ is a measurable function ( $A$ is a compact subset of some metric space), $b_{i} \in L^{\infty}\left(\mathbb{T}^{N} \times A ; \mathbb{R}^{N}\right)$, satisfies

$$
\left|b_{i}(x, a)-b_{i}(y, a)\right| \leq C|x-y|, \quad x, y \in \mathbb{T}^{N}, a \in A, 1 \leq i \leq m .
$$

For every $a_{t}$ and matrix of probability transition $G=\left(\gamma_{i j}\right)_{i, j}$ satisfying $\sum_{j \neq i} \gamma_{i j}=1$ for $i \neq j$ and $\gamma_{i i}=-1$, there exists a solution $\left(X_{t}, \nu_{t}\right)$, where $X_{t}:[0, \infty) \rightarrow \mathbb{T}^{N}$ is piecewise $C^{1}$ and $\nu(t)$ is a continuous-time Markov chain with state space $\{1, \ldots, m\}$ and probability transitions given by

$$
\mathbb{P}\left\{\nu_{t+\Delta t}=j \mid \nu_{t}=i\right\}=\gamma_{i j} \Delta t+o(\Delta t)
$$

for $j \neq i$.

We introduce the value functions of the optimal control problems

$$
u_{i}(x, t)=\inf _{a_{t} \in L^{\infty}([0, t], A)} \mathbb{E}_{x, i}\left\{\int_{0}^{t} f_{\nu_{s}}\left(X_{s}\right) d s+u_{0, \nu_{t}}\left(X_{t}\right)\right\}, \quad i=1, \ldots m,
$$

where $\mathbb{E}_{x, i}$ denote the expectation of a trajectory starting at $x$ in the mode $i$, $f_{i}, u_{0, i}: \mathbb{T}^{N} \rightarrow \mathbb{R}$ are continuous and $f_{i} \geq 0$.

It is possible to show that the following dynamic programming principle holds:

$$
u_{i}(x, t)=\inf _{a_{t} \in L^{\infty}([0, t], A)} \mathbb{E}_{x, i}\left\{\int_{0}^{t} f_{\nu_{s}}\left(X_{s}\right) d s+u_{\nu_{h}}\left(X_{h}, t-h\right)\right\} \quad 0<h \leq t .
$$

Then the functions $u_{i}$ satisfy the system

$$
\left\{\begin{array}{lc}
\frac{\partial u_{i}}{\partial t}+\sup _{a \in A}-\left\langle b_{i}(x, a), D u_{i}\right\rangle+\sum_{j \neq i} \gamma_{i j}\left(u_{i}-u_{j}\right)=f_{i} & (x, t) \in \mathbb{T}^{N} \times(0,+\infty), \\
i=1, \ldots m, & x \in \mathbb{T}^{N},
\end{array}\right.
$$

which has the form (5.1) by setting $F_{i}(x, p)=\sup _{a \in A}-\left\langle b_{i}(x, a), p\right\rangle$ and $d_{i i}=$ $\sum_{j \neq i} \gamma_{i j}=1$ and $d_{i j}=-\gamma_{i j}$ for $j \neq i$.

The assumptions (2.1), (3.3) and (3.5) are clearly satisfied and (3.4) holds if the following controllability assumption is satisfied: for every $i$, there exists $r>0$ such that for any $x \in \mathbb{T}^{N}$, the ball $B(0, r)$ is contained in $\overline{\operatorname{co}}\left\{b_{i}(x, A)\right\}$. Moreover, $\cap_{1 \leq i \leq m} \mathcal{D}_{i}=\mathbb{T}^{N}$ and $\mathcal{A}=\mathcal{F}$.

Assuming (3.7), (2.4), we obtain that Theorem 5.4 holds. Roughly speaking, it means that the optimal strategy is to drive the trajectories towards a point $x^{*}$ of $\mathcal{A}$ and then not to move anymore (except maybe a small time before $t$ ). This is suggested by the fact that all the $f_{i}$ 's have minimum 0 at $x^{*}$ and, at such point, the running cost is 0 . Now, if (3.7) does not hold anymore, things appear to be more complicated. We hope to come back to this issue in a future work.

\section{Acknowledgments}

O.L. is partially supported by the project ANR BLANC07-3 187245, "Hamilton-Jacobi and Weak KAM Theory". We would like to thank G. Barles 
and P. Cardaliaguet who helped us to improve the first version of this work and M. Briane, L. Hervé and J. Ledoux for useful references and suggestions.

\section{References}

[1] Bardi, M., Capuzzo Dolcetta, I.: Optimal Control and Viscosity Solutions of Hamilton-Jacobi-Bellman Equations. Birkhäuser Boston Inc., Boston (1997)

[2] Barles, G.: Solutions de viscosité des équations de Hamilton-Jacobi. Springer, Paris (1994)

[3] Barles, G., Roquejoffre, J.-M.: Ergodic type problems and large time behaviour of unbounded solutions of Hamilton-Jacobi equations. Commun. Partial Differ. Equ. 31(7-9), 1209-1225 (2006)

[4] Barles, G., Souganidis, P.E.: On the large time behavior of solutions of Hamilton-Jacobi equations. SIAM J. Math. Anal. 31(4), 925-939 (2000)

[5] Berman, A., Plemmons, R.J.: Nonnegative matrices in the mathematical sciences. In: Classics in Applied Mathematics, vol. 9. Society for Industrial and Applied Mathematics (SIAM), Philadelphia (1994). Revised reprint of the 1979 original

[6] Busca, J., Sirakov, B.: Harnack type estimates for nonlinear elliptic systems and applications. Ann. Inst. H. Poincaré Anal. Non Linéaire 21(5), 543-590 (2004)

[7] Camilli, F., Loreti, P.: Comparison results for a class of weakly coupled systems of eikonal equations. Hokkaido Math. J. 37, 349-362 (2008)

[8] Camilli, F., Ley, O., Loreti, P.: Homogenization of monotone systems of Hamilton-Jacobi equations. ESAIM Control Optim. Calc. Var. 16, 58-76 (2010)

[9] Davini, A., Siconolfi, A.: A generalized dynamical approach to the large time behavior of solutions of Hamilton-Jacobi equations. SIAM J. Math. Anal. 38(2), 478-502 (2006)

[10] Engler, H., Lenhart, S.M.: Viscosity solutions for weakly coupled systems of Hamilton-Jacobi equations. Proc. Lond. Math. Soc. (3) 63(1), 212-240 (1991)

[11] Fathi, A.: Sur la convergence du semi-groupe de Lax-Oleinik C. R. Acad. Sci. Paris Sér. I Math. 327(3), 267-270 (1998)

[12] Fathi, A.: Weak KAM theorem in Lagrangian dynamics. In: Cambridge Studies in Advanced Mathematics, vol. 88. Cambridge University Press, Cambridge (2010)

[13] Fathi, A., Siconolfi, A.: PDE aspects of Aubry-Mather theory for quasiconvex Hamiltonians. Calc. Var. Partial Differ. Equ. 22(2), 185-228 (2005)

[14] Fleming, W.H., Zhang, Q.: Risk-sensitive production planning of a stochastic manufacturing system. SIAM J. Control Optim. 36(4), 1147-1170 (1998) 
[15] Ichihara, N., Ishii, H.: Long-time behavior of solutions of Hamilton-Jacobi equations with convex and coercive Hamiltonians. Arch. Ration. Mech. Anal. 194(2), 383-419 (2009)

[16] Ishii, H.: Perron's method for monotone systems of second-order elliptic partial differential equations. Differ. Integr. Equ. 5(1), 1-24 (1992)

[17] Ishii, H.: Asymptotic solutions for large time of Hamilton-Jacobi equations. In: International Congress of Mathematicians, vol. III, pp. 213-227. Eur. Math. Soc., Zürich (2006)

[18] Ishii, H.: Asymptotic solutions for large time of Hamilton-Jacobi equations in Euclidean $n$ space. Ann. Inst. H. Poincaré Anal. Non Linéaire 25(2), 231-266 (2008)

[19] Ishii, H.: Asymptotic solutions of Hamilton-Jacobi equations for large time and related topics. In: ICIAM 07-6th International Congress on Industrial and Applied Mathematics, pp. 193-217. Eur. Math. Soc., Zürich (2009)

[20] Ishii, H., Koike, S.: Remarks on elliptic singular perturbation problems. Appl. Math. Optim. 23(1), 1-15 (1991)

[21] Mitake, H.: The large-time behavior of solutions of the Cauchy-Dirichlet problem for Hamilton-Jacobi equations. Nonlinear Differ. Equ. Appl. 15(3), 347-362 (2008)

[22] Mitake, H.: Asymptotic solutions of Hamilton-Jacobi equations with state constraints. Appl. Math. Optim. 58(3), 393-410 (2008)

[23] Mitake, H.: Large time behavior of solutions of Hamilton-Jacobi equations with periodic boundary data. Nonlinear Anal. 71(11), 5392-5405 (2009)

[24] Namah, G., Roquejoffre, J.-M.: Remarks on the long time behaviour of the solutions of Hamilton-Jacobi equations. Commun. Partial Differ. Equ. 24(5-6), 883$893(1999)$

[25] Roquejoffre, J.-M.: Convergence to steady states or periodic solutions in a class of Hamilton-Jacobi equations. J. Math. Pures Appl. (9) 80(1), 85-104 (2001)

[26] Seneta, E.: Nonnegative matrices and Markov chains. In: Springer Series in Statistics, 2nd edn. Springer, New York (1981)

[27] Yin, G.G., Zhang, Q.: Continuous-time Markov chains and applications. In: Applications of Mathematics, vol. 37. A Singular Perturbation Approach. Springer, New York (1998)

Fabio Camilli and Paola Loreti

Dipartimento di Scienze di Base e Applicate per l'Ingegneria

"Sapienza" Università di Roma

00161 Rome

Italy

e-mail: camilli@dmmm.uniroma1.it 
Paola Loreti

e-mail: loreti@dmmm.uniroma1.it

Olivier Ley and Vinh Duc Nguyen

IRMAR

INSA de Rennes

35708 Rennes

France

e-mail: olivier.ley@insa-rennes.fr

Vinh Duc Nguyen

e-mail: vinh.nguyen@insa-rennes.fr

Received: 1 September 2011.

Accepted: 3 December 2011. 